\title{
Measurements of differential cross sections of top quark pair production as a function of kinematic event variables in proton-proton collisions at $\sqrt{s}=13 \mathrm{TeV}$
}

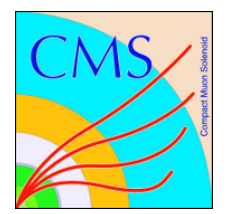

\section{The CMS collaboration}

E-mail: cms-publication-committee-chair@cern.ch

AbSTRACT: Measurements of differential t $t \bar{t}$ production cross sections are presented in the single-lepton decay channel, as a function of a number of kinematic event variables. The measurements are performed with proton-proton collision data at $\sqrt{s}=13 \mathrm{TeV}$, collected by the CMS experiment at the LHC during 2016, with an integrated luminosity of $35.9 \mathrm{fb}^{-1}$. The data are compared to a variety of state-of-the-art leading-order and next-to-leadingorder $\mathrm{t} \overline{\mathrm{t}}$ simulations.

KEYwords: Hadron-Hadron scattering (experiments), Top physics

ArXiv EPrint: 1803.03991 


\section{Contents}

1 Introduction $\quad 1$

2 The CMS detector 2

3 Signal sample and background simulation 3

4 Event reconstruction and selection 4

5 Cross section measurement $\quad 5$

$\begin{array}{lll}5.1 & \text { Particle level and visible phase space definitions } & 6\end{array}$

$\begin{array}{ll}5.2 \text { Unfolding and cross section calculation } & 8\end{array}$

$\begin{array}{llr}6 & \text { Systematic uncertainties } & 9\end{array}$

$\begin{array}{lll}7 & \text { Cross section results } & 13\end{array}$

$\begin{array}{lll}8 & \text { Summary } & 24\end{array}$

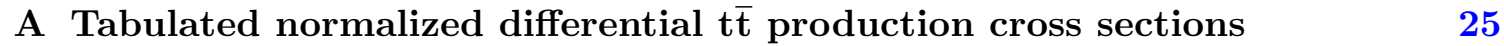

B Tabulated absolute differential $t \overline{\mathrm{t}}$ production cross sections $\quad 29$

C Tabulated minimum and maximum relative uncertainties for absolute cross sections $\quad 32$

$\begin{array}{lr}\text { The CMS collaboration } & 38\end{array}$

\section{Introduction}

In 2016 the CERN LHC collided protons at $\sqrt{s}=13 \mathrm{TeV}$, resulting in a data set recorded by the CMS experiment [1], with an integrated luminosity of $35.9 \mathrm{fb}^{-1}$. Approximately 30 million top quark-antiquark pairs $(\mathrm{t} \overline{\mathrm{t}})$ are present in this data set, which allows detailed studies of the production properties of $t \bar{t}$ events to be performed.

Measurements of kinematic distributions in $t \bar{t}$ events are important for verifying current

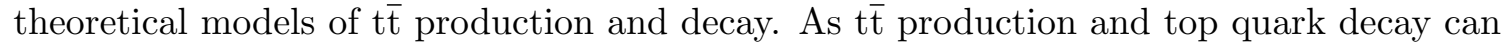
be a significant source of background events in many searches for physics beyond the standard model, for example in searches for supersymmetric models with top-quark-like signatures, it is important that $t \bar{t}$ production be well understood and modeled. In addition to physics beyond the standard model, a good understanding of $t \bar{t}$ production is necessary 


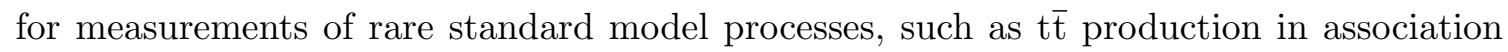
with a W, Z, or Higgs boson.

In this paper, we present measurements of differential t $\overline{\mathrm{t}}$ production cross sections, as a function of kinematic event variables that do not require the reconstruction of the $t \bar{t}$ system. Events are considered when the final state includes exactly one isolated lepton $(\ell=\mathrm{e}$ or $\mu)$ with large transverse momentum $p_{\mathrm{T}}$ and at least four jets, of which at least two are tagged as originating from a bottom (b) quark. The kinematic event variables are the jet multiplicity $\left(N_{\text {jets }}\right)$, the scalar sum of the jet $p_{\mathrm{T}}\left(H_{\mathrm{T}}\right)$, the scalar sum of the $p_{\mathrm{T}}$ of all particles $\left(S_{\mathrm{T}}\right)$, the transverse momentum imbalance $\left(p_{\mathrm{T}}^{\text {miss }}\right)$, the magnitude of the $p_{\mathrm{T}}$ of the leptonically decaying $\mathrm{W}$ boson $\left(p_{\mathrm{T}}^{\mathrm{W}}\right)$, and the magnitudes of the $p_{\mathrm{T}}$ and pseudorapidity of the lepton $\left(p_{\mathrm{T}}^{\ell}\right.$ and $\left.\left|\eta^{\ell}\right|\right)$.

The measurements of the differential t $\bar{t}$ production cross sections are presented at particle level, i.e. with respect to generated "stable" particles (with a mean lifetime longer than $30 \mathrm{ps}$ ), in a phase space that closely resembles that accessible by the CMS detector (the visible phase space). This avoids the influence of large theoretical uncertainties that would be introduced by extrapolating the measurements to a larger phase space, or by presenting the measurements at parton level.

Several measurements of the differential t $t \bar{t}$ production cross sections as a function of the properties of the $t \bar{t}$ system and of the jet activity in $t \bar{t}$ events have been performed at the $\mathrm{LHC}$, at 7 and $8 \mathrm{TeV}[2-6]$, and $13 \mathrm{TeV}$ [7-10]. Measurements with respect to kinematic event variables in $t \bar{t}$ events have been performed with the CMS detector at 7 and $8 \mathrm{TeV}$ [11].

\section{The CMS detector}

The central feature of the CMS apparatus is a superconducting solenoid of $6 \mathrm{~m}$ internal diameter, providing a magnetic field strength of $3.8 \mathrm{~T}$. Within the solenoid volume are a silicon pixel and strip tracker, a lead tungstate crystal electromagnetic calorimeter (ECAL), and a brass and scintillator hadron calorimeter, each composed of a barrel and two endcap sections. Forward calorimeters extend the $\eta$ coverage provided by the barrel and endcap detectors. Muons are measured in gas-ionization detectors embedded in the steel fluxreturn yoke outside the solenoid. A more detailed description of the CMS detector, together with a definition of the coordinate system used and the relevant kinematic variables, can be found in ref. [1].

Events of interest are selected using a two-tiered trigger system [12]. The first level, composed of custom hardware processors, uses information from the calorimeters and muon detectors to select events at a rate of around $100 \mathrm{kHz}$ within a time interval of less than $4 \mu \mathrm{s}$. The second level, known as the high-level trigger (HLT), consists of a farm of processors running a version of the full event reconstruction software optimized for fast processing, and reduces the event rate to around $1 \mathrm{kHz}$ before data storage. 


\section{Signal sample and background simulation}

Two independent $t \bar{t}$ samples are simulated with the POWHEG (v2) generator [13-16], which utilizes next-to-leading-order (NLO) matrix-element calculations. One sample uses PYTHIA (v8.212) [17, 18] with the CUETP8M2T4 tune [19] for the simulation of the parton shower and hadronization. The second has parton showering and hadronization performed by HERWIG $++(2.7 .1)[20]$ using the tune EE5C [21].

Two additional independent simulated $t \overline{\mathrm{t}}$ samples are produced with the MG5_aMC@NLO (v2.2.2) generator [22]. In the first, MG5_aMC@NLO is used to generate events at leading-order (LO) accuracy with up to three additional partons, and PYTHIA is employed with the CUETP8M1 tune [23] for parton showering and hadronization. The MLM jet-parton matching algorithm [24] is used in this sample, referred to as MG5_aMC@NLO-LO. In the second, MG5_aMC@NLO simulates events to NLO accuracy with up to two additional partons, where parton showering and hadronization are performed using PYTHIA with the CUETP8M2T4 tune. The FxFx jet-parton matching algorithm [25] is used, and this sample is referred to as MG5_aMC@NLO-NLO. It is important to compare multiple $t \bar{t}$ generators in order to find the current most suitable description of top quark production and decay, and to identify any discrepancies in the models.

In all simulated $t \bar{t}$ samples, the top quark mass is set to $172.5 \mathrm{GeV}$. The NNPDF30_nlo_as_0118 parton distribution function (PDF) set is used for the NLO samples while the NNPDF30_lo_as_0130 set is used for the LO samples [26]. When comparing with reconstructed data, a cross section of $832_{-29}^{+20}$ (scale) $\pm 35\left(\mathrm{PDF}+\alpha_{S}\right) \mathrm{pb}$ is used to normalize the $t \bar{t}$ samples, where $\alpha_{S}$ is the strong coupling constant. This t $\bar{t}$ cross section is calculated to next-to-next-to-leading-order (NNLO) accuracy in quantum chromodynamics (QCD) including resummation of next-to-next-to-leading logarithmic soft-gluon terms with Top ++ (v2.0) [27-33]. The scale uncertainty in this t $\bar{t}$ cross section comes from the independent variation of the factorization and renormalization scales.

The dominant background processes to t $\overline{\mathrm{t}}$ production, i.e. the production of single top quarks and the production of vector bosons in association with jets, are also simulated. Single top quark processes are generated with POWHEG interfaced with PYTHIA, and are normalized to cross sections that are calculated to NLO precision [34, 35]. Separate samples are generated for $t$ - and $s$-channel production [36, 37]. The sample of single top quarks in association with a W boson is produced with POWHEG (v1) [38]. In this sample, the diagram removal scheme [39] is used to avoid double counting of Feynman diagrams in the production of single top quarks in association with a $\mathrm{W}$ boson at NLO and top quark pair production. Samples of $\mathrm{W}$ and $\mathrm{Z}$ boson production with leptonic final states, in association with jets $(\mathrm{V}+\mathrm{jets})$, are generated with MG5_aMC@NLO-LO. Separate samples are generated with exactly one, two, three, and four additional jets to ensure a large sample of events that are likely to mimic the signature of tét production. These samples are normalized to their NNLO cross sections [40].

In addition, QCD multijet events are generated with PYTHIA for matrix-element calculations, parton shower simulation, and hadronization. To obtain a large sample of QCD multijet events that are likely to mimic the signature of t $\bar{t}$ production in the single-lepton 
decay channel, only events with large electromagnetic activity or containing a muon are generated. These samples are normalized to their LO cross sections and are used to create transfer factors from a control region to the signal region for a QCD background estimate based on data in the control region. The CMS detector response for all simulated samples is modeled using GEANT4 [41].

\section{Event reconstruction and selection}

Parallel selection paths are defined to target $t \bar{t}$ events that decay to final states containing an electron (e+jets) or a muon $(\mu+$ jets). The HLT in the e+jets channel requires at least one isolated electron candidate with $p_{\mathrm{T}}>32 \mathrm{GeV}$ and $|\eta|<2.1$. The corresponding requirements in the $\mu+$ jets channel are at least one isolated muon candidate with $p_{\mathrm{T}}>$ $24 \mathrm{GeV}$ and $|\eta|<2.4$.

Offline reconstruction and selection uses the particle-flow (PF) algorithm [42] to reconstruct and identify each individual particle with an optimized combination of information from the subdetectors of CMS. In the e+jets channel, electron candidates are required to satisfy $p_{\mathrm{T}}>34 \mathrm{GeV}$ and $|\eta|<2.1$. Electron candidates whose energy deposition in the ECAL is in the transition region between the barrel and endcap regions of the ECAL are not considered due to less efficient electron reconstruction. Electron candidates must also satisfy several identification criteria [43] to suppress the rate of jets and converted photons that are identified incorrectly as electron candidates. In addition, electron candidates must be isolated. To calculate the isolation, a cone of size $\Delta R=\sqrt{(\Delta \eta)^{2}+(\Delta \phi)^{2}}=0.3$ is constructed around the electron direction, where $\phi$ is the azimuthal angle. The sum of the $p_{\mathrm{T}}$ of all PF candidates within this cone is calculated, excluding the lepton candidate and is corrected for the effects of additional proton-proton collisions within the same or nearby bunch crossings. The relative isolation variable $I_{\text {rel }}$ is defined as the ratio of this sum to the electron $p_{\mathrm{T}}$, and is required to be less than $6 \%$.

In the $\mu+$ jets channel, muon candidates are required to satisfy $p_{\mathrm{T}}>26 \mathrm{GeV}$ and $|\eta|<2.4$. Similarly to the electron candidates, muon candidates must satisfy additional identification criteria [44]. Muon candidates must be isolated, satisfying $I_{\text {rel }}<15 \%$ where $I_{\text {rel }}$ is defined as for electrons, but with a cone of size $\Delta R=0.4$.

For both electron and muon candidates, the lepton must be associated with the primary interaction vertex of the event. The primary interaction vertex is defined as the reconstructed vertex associated with the largest sum of $p_{T}^{2}$ from physics objects that have been defined using information from the tracking detector, including jets, the associated missing transverse momentum, which was taken as the negative vector sum of the $p_{\mathrm{T}}$ of those jets, and charged leptons.

The trigger, reconstruction and identification efficiencies for both electrons and muons are measured in data, and corrected in simulation to match those seen in data. The efficiencies are calculated using the tag-and-probe method [45] from events containing a Z boson. The total lepton correction factors are between 0.95 and 1 .

Jets are clustered from PF candidates with the anti- $k_{\mathrm{T}}$ algorithm [46] implemented in the FASTJET package [47], with a distance parameter of 0.4. The jet momentum is deter- 
mined as the vector sum of the $p_{\mathrm{T}}$ of all PF candidates in the jet. A correction is applied to jet energies to take into account the contribution from additional proton-proton interactions using the charged hadron subtraction method [48]. The measured energy of each jet is corrected for known variations in the jet energy response as a function of the measured jet $\eta$ and $p_{\mathrm{T}}$. The jet energy resolution (JER) is corrected in simulation to match that seen in data. Jets are required to satisfy $p_{\mathrm{T}}>30 \mathrm{GeV}$ and $|\eta|<2.4$. Jets closer than $\Delta R=0.4$ to identified isolated leptons are removed, as they are likely to have originated from the lepton itself.

The combined secondary vertex algorithm $[49,50]$ is used to identify jets originating from a b quark. The threshold of the algorithm is chosen such that the identification efficiency (in simulation) of genuine b quark jets is $\approx 70 \%$, and the probability to mistag a light quark or gluon jet is $\approx 1 \%$. The identification efficiency of $\mathrm{b}$ quark jets in simulation is corrected to match that seen in data.

The distribution of the number of additional proton-proton interactions in simulation is corrected to match data. Events must contain exactly one high- $p_{\mathrm{T}}$, isolated electron or muon. Events are vetoed if they contain an additional isolated lepton candidate with $p_{\mathrm{T}}>15 \mathrm{GeV}$ and $|\eta|<2.4$. Events must also contain at least four jets, at least two of which are required to be identified as originating from a $b$ quark.

\section{Cross section measurement}

As stated in section 1 , the differential t $\bar{t}$ production cross sections are measured as a function of the kinematic event variables: $N_{\text {jets }}, H_{\mathrm{T}}, S_{\mathrm{T}}, p_{\mathrm{T}}^{\mathrm{miss}}, p_{\mathrm{T}}^{\mathrm{W}}, p_{\mathrm{T}}^{\ell}$ and $\left|\eta^{\ell}\right|$. The $N_{\text {jets }}$ variable is the total number of jets in the event with $p_{\mathrm{T}}>30 \mathrm{GeV}$ and $|\eta|<2.4$. The variable $H_{\mathrm{T}}$ is the scalar sum of the $p_{\mathrm{T}}$ of these jets. The quantity $p_{\mathrm{T}}^{\text {miss }}$ is defined as the magnitude of $\vec{p}_{\mathrm{T}}^{\text {miss }}$, the transverse projection of the negative vector sum of the momenta of all reconstructed PF candidates in an event. The $p_{\mathrm{T}}^{\ell}$ and $\left|\eta^{\ell}\right|$ variables are magnitudes of the transverse momentum and the pseudorapidity of the lepton in the event, respectively. The variable $S_{\mathrm{T}}$ is the sum of $H_{\mathrm{T}}, p_{\mathrm{T}}^{\text {miss }}$, and $p_{\mathrm{T}}^{\ell}$. The variable $p_{\mathrm{T}}^{\mathrm{W}}$ is the magnitude of the transverse momentum of the leptonically decaying $\mathrm{W}$ boson, which is constructed from $\vec{p}_{\mathrm{T}}^{\ell}$ and $\vec{p}_{\mathrm{T}}^{\text {miss }}$.

The distributions of these variables measured in data are shown in figures 1 and 2, and are compared to the sum of signal and background events from simulation. A total of 662381 events are measured in data, of which $92.1 \%$ are predicted from the POWHEG+PYTHIA simulation to be $\mathrm{t} \overline{\mathrm{t}}$ events. Single top quark production and V+jets production contribute $4.4 \%$ and $2.1 \%$ to the total number of events, respectively, as estimated from simulation. The component of multijet QCD events is estimated from control regions in the data, and comprises approximately $1.4 \%$ of the total number of events. The control regions are designed to obtain data samples that are enriched in QCD multijet events that are kinematically similar to the signal region, but with little contamination from $\mathrm{t} \overline{\mathrm{t}}$, single top quark, and $\mathrm{V}+$ jets events. In the e+jets channel, the control region is obtained by inverting the isolation criterion on electron candidates. In the $\mu+$ jets channel, the control region is obtained by requiring muon candidates to satisfy $0.15<I_{\text {rel }}<0.30$. In the control regions for both channels, the number of b-tagged jets is also required to be exactly zero. The contribution of $t \bar{t}$, single top quark and $V+$ jets events to the control 
regions $(\approx 15-20 \%)$ is estimated from simulation with all corrections and subtracted from the data. The ratio of the number of multijet QCD events in the control region to that in the signal region (the transfer factor), both predicted from simulation, is then used to scale the normalization of the data control region to obtain the multijet QCD estimate in the signal region. Other sources of background are negligible, and are not considered in this measurement. The level of agreement between the total event count of data and simulation, within $0.2 \%$, indicates that the total cross section is compatible to that stated in section 3 .

Previous measurements [2-8] report that the top quark $p_{\mathrm{T}}$ spectrum in data is softer than that predicted by NLO simulation. This effect can be seen in some of the distributions in figures 1 and 2, where distributions correlated with the top quark $p_{\mathrm{T}}$ are also softer in data than those predicted by the simulation.

\subsection{Particle level and visible phase space definitions}

The results are presented at particle level, i.e. with respect to the stable particles produced in simulation by the event generator, before detector interactions are modeled. The generator-level definitions for the particles and visible phase space are based on the RIVET framework [51], following the prescriptions adopted in ref. [52]. Generated electrons and muons not originating from a hadron or a quark are used to define electrons and muons at particle level. Photons that are near the lepton are assumed to have radiated from it, and are clustered together with the anti- $k_{\mathrm{T}}$ algorithm with a distance parameter of 0.1 .

Particle-level jets are constructed by clustering all stable particles, excluding the lepton, with the anti- $k_{\mathrm{T}}$ algorithm using a distance parameter of 0.4. To determine if a particle-level jet originated from a b quark, b hadrons are included in the clustering of jets, but with the magnitude of the four-momentum of the $\mathrm{b}$ hadron scaled to a negligible value. The $b$ hadrons can then be clustered into jets without affecting the kinematic properties of the jet. A jet with a b hadron among its constituents is considered to have originated from a b quark. The particle-level $p_{\mathrm{T}}^{\text {miss }}$ is calculated from all stable visible particles.

The differential t $t \bar{t}$ production cross sections are measured in a visible phase space, which is chosen to be the same for both e+jets and $\mu+$ jets channels, and to closely resemble the criteria used to select events in data. Particle-level objects are used to define the common visible phase space of $\mathrm{t} \overline{\mathrm{t}}$ events for both $\mathrm{e}+\mathrm{jets}$ and $\mu+$ jets channels, all within $|\eta|<2.4$, which requires exactly one electron or muon with $p_{\mathrm{T}}>26 \mathrm{GeV}$, and no additional electrons or muons with $p_{\mathrm{T}}>15 \mathrm{GeV}$. The event must also contain at least three particlelevel jets with $p_{\mathrm{T}}>30 \mathrm{GeV}$, and one jet with $p_{\mathrm{T}}>20 \mathrm{GeV}$. Two of these particle-level jets must also be tagged as originating from a b quark. The $H_{\mathrm{T}}, S_{\mathrm{T}}$, and $N_{\text {jets }}$ variables are calculated at the particle level with respect to all particle-level jets with $p_{\mathrm{T}}>20 \mathrm{GeV}$ and $|\eta|<2.4$. This choice of particle-level phase space is made to obtain the largest possible data sample, and the uncertainty in the resulting extrapolation makes only a small contribution to the uncertainty in the final results.

The yield of $t \bar{t}$ events for each bin in data is obtained by subtracting the contribution of each background process. The contribution of $t \bar{t}$ events that satisfy the selection criteria, but do not enter the visible phase space at particle level, is estimated from simulation and also subtracted from the data. This amounts to approximately $7 \%$ of all $t \bar{t}$ events and are 

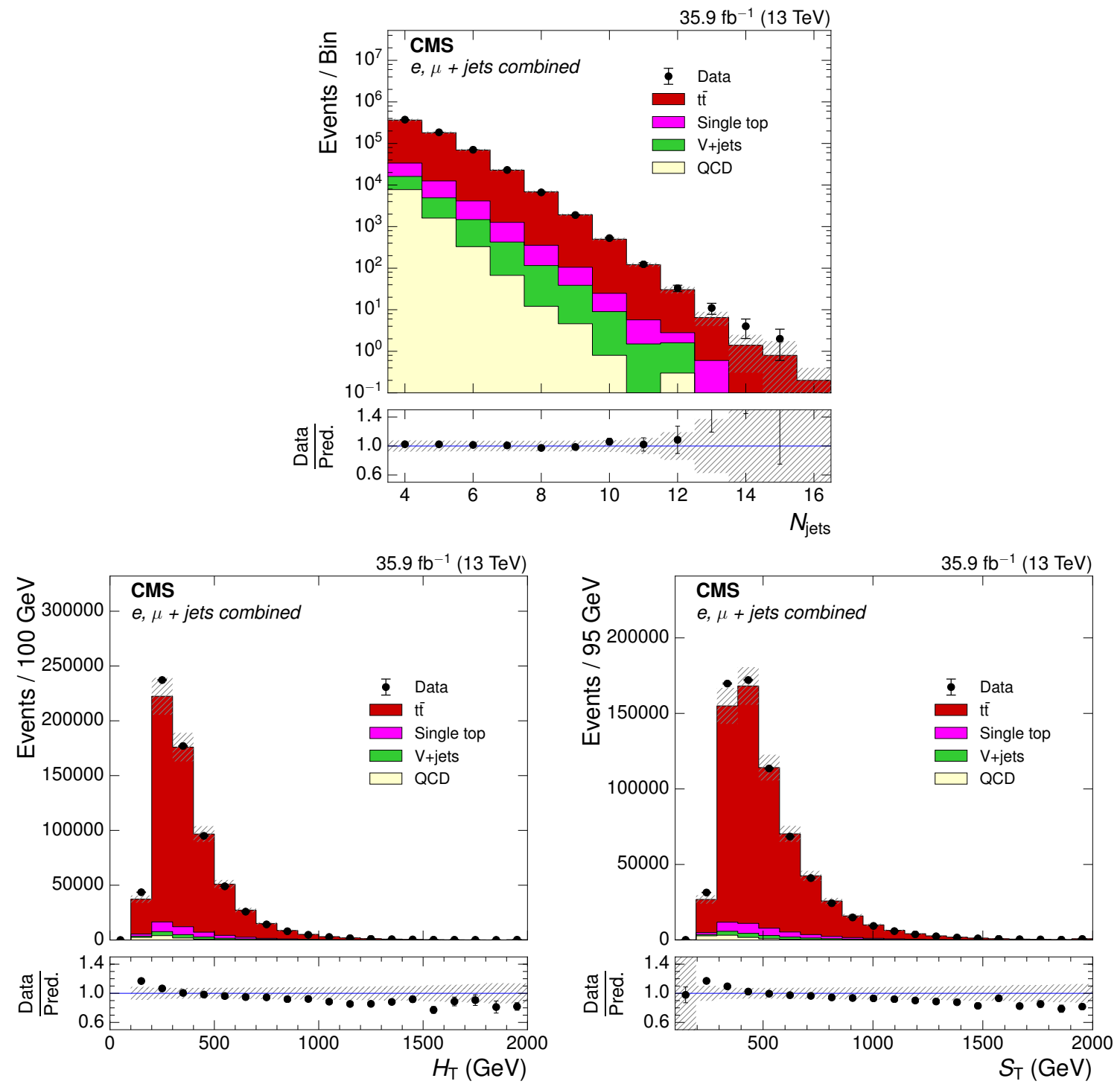

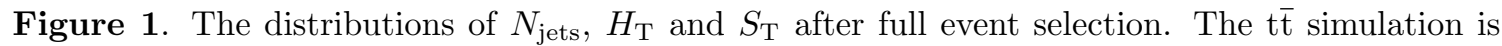
normalized to the NNLO prediction. The ratio of the number of events in data to that in simulation is shown below each of the distributions, with the statistical uncertainty in the data shown by the vertical uncertainty bars. The statistical uncertainty in the number of simulation events and the uncertainties in the modeling in simulation are shown by the hatched band.

predominately those in which one of the jets fails the particle-level jet selection, but passes the reconstructed jet selection because of the resolution of the detector. No selection is applied on the decay channel of the top quarks, so the phase space does not exclusively contain semileptonic (electron or muon) t $\overline{\mathrm{t}}$ events. In particular, there are contributions from events where one top quark decays to a tau lepton and subsequently to an electron or muon, or where both top quarks decay leptonically but one lepton is not within the acceptance. 

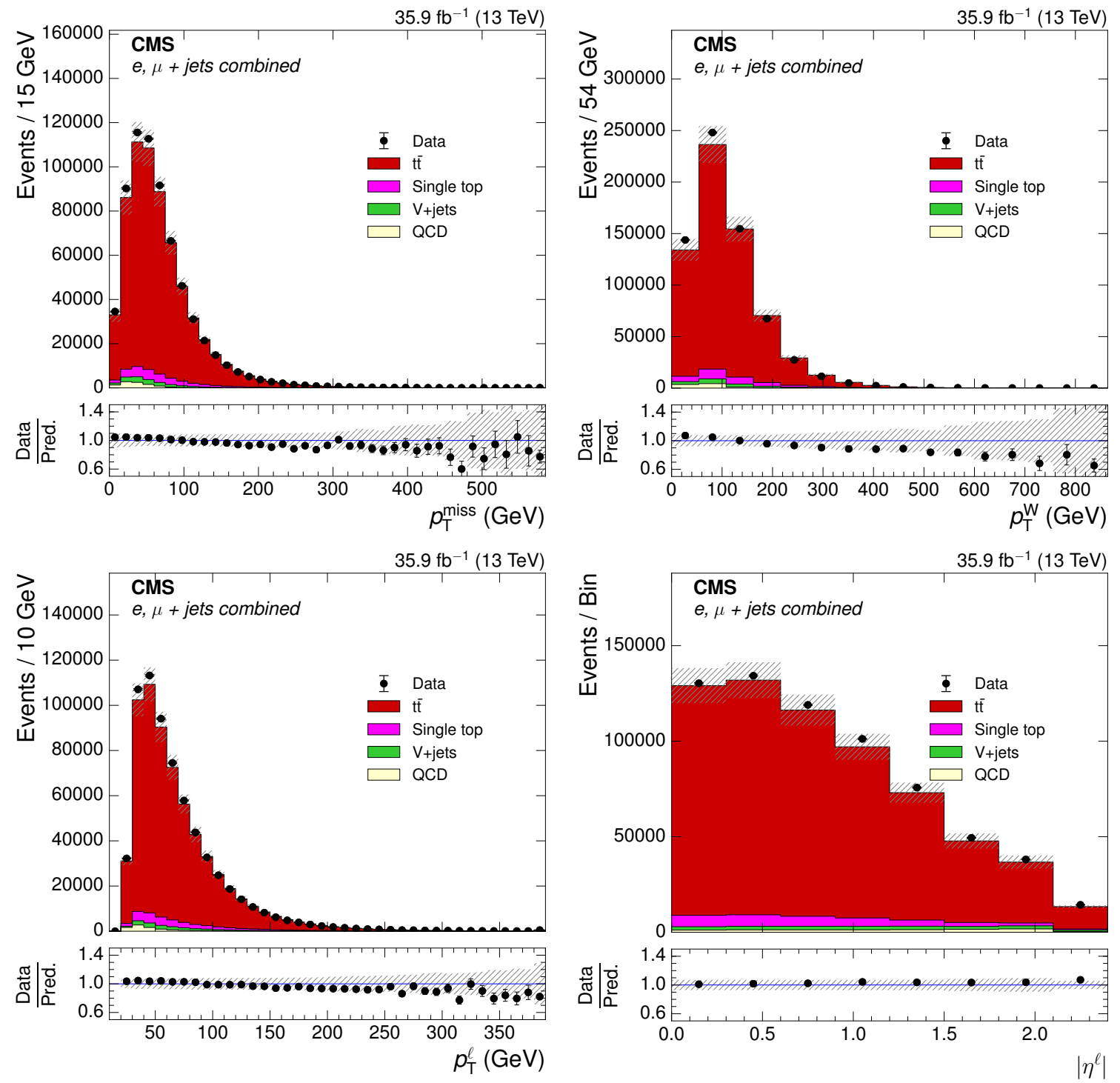

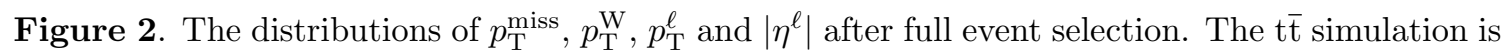
normalized to the NNLO prediction. The ratio of the number of events in data to that in simulation is shown below each of the distributions, with the statistical uncertainty in the data shown by the vertical uncertainty bars. The statistical uncertainty in the number of simulation events and the uncertainties in modeling in simulation are shown by the hatched band.

\subsection{Unfolding and cross section calculation}

For each kinematic event variable the yield of $t \bar{t}$ events in each bin is unfolded to correct for the detector acceptance, efficiency, and bin-to-bin migrations stemming from the detector resolution to obtain the yield of $t \bar{t}$ events in the visible phase space at the particle level. The bin widths are chosen to give a low level of bin-to-bin migration, and are always greater than the detector resolution. 
A response matrix, constructed using the POWHEG+PYTHIA sample, relates the kinematic event distributions at reconstruction level to those at particle level. The response matrix also includes efficiency and acceptance corrections. Unfolding is performed by inverting the response matrix, based on a least-squares fit with Tikhonov regularization, implemented in the TUNFOLD software framework [53]. Regularization dampens nonphysical fluctuations in the unfolded $t \bar{t}$ yields, and the regularization parameter is chosen by minimizing the average global statistical correlation between the bins of each variable. The typical regularization parameters are found to be of order $10^{-4}-10^{-3}$, and significantly lower for the $\left|\eta^{\ell}\right|$ variable.

The yields of $t \bar{t}$ events are unfolded separately in the $\mathrm{e}+\mathrm{jets}$ and $\mu+$ jets channels and then combined after unfolding, giving the total number of t $\bar{t}$ events at particle level in the visible phase space, $N_{\mathrm{t} \overline{\mathrm{t}}}$. The normalized differential cross section with respect to each variable, $X$, can then be calculated using

$$
\frac{1}{\sigma_{\mathrm{t} \overline{\mathrm{t}}}^{\mathrm{vis}}} \frac{\mathrm{d} \sigma_{\mathrm{t} \overline{\mathrm{t}}}^{i}}{\mathrm{~d} X}=\frac{1}{\sum_{j} N_{\mathrm{t} \overline{\mathrm{t}}}^{j}} \frac{N_{\mathrm{t} \overline{\mathrm{t}}}^{i}}{\Delta X^{i}},
$$

where $\sigma_{\mathrm{t} \overline{\mathrm{t}}}^{\mathrm{vis}}$ is the total $\mathrm{t} \overline{\mathrm{t}}$ production cross section in the visible phase space, $\sigma_{\mathrm{t} \overline{\mathrm{t}}}^{i}$ is the $\mathrm{t} \overline{\mathrm{t}}$ production cross section in bin $i, N_{\mathrm{t} \overline{\mathrm{t}}}^{i(j)}$ is the number of $\mathrm{t} \overline{\mathrm{t}}$ events in bin $i(j)$ after unfolding, and $\Delta X^{i}$ is the width of bin $i$. The absolute differential cross section can be calculated as

$$
\frac{\mathrm{d} \sigma_{\mathrm{t} \overline{\mathrm{t}}}^{i}}{\mathrm{~d} X}=\frac{N_{\mathrm{t} \overline{\mathrm{t}}}^{i}}{\mathcal{L} \Delta X^{i}}
$$

where $\mathcal{L}$ is the integrated luminosity of the data.

\section{Systematic uncertainties}

Sources of systematic uncertainties are evaluated and propagated to the final result by recalculating the response matrix with a modified $t \bar{t}$ simulation and/or by modifying the background predictions.

The uncertainty in the integrated luminosity of the data is estimated to be $\pm 2.5 \%$ [54]. The uncertainty in the number of additional inelastic interactions in the same or nearby bunch crossings is estimated by varying the total proton-proton inelastic cross section by $\pm 4.6 \%$ [55]. This cross section is used in determining the distribution of additional inelastic interactions in data, which is used to correct the simulation.

The uncertainty in the efficiency of the b quark jet identification and mistagging rate in the simulation is taken as the uncertainty in the $p_{\mathrm{T}},|\eta|$, and flavor-dependent correction factors [50]. The uncertainties in the lepton trigger, reconstruction, and identification correction factors are similarly propagated to the final results.

The uncertainties in the jet energy scale (JES) and JER are estimated as functions of jet $p_{\mathrm{T}}$ and $|\eta|$ [48]. The uncertainty in the JES is also propagated into the calculation of $p_{\mathrm{T}}^{\text {miss }}$. Additional uncertainties in the $p_{\mathrm{T}}$ of electrons, muons, tau leptons and other unclustered PF candidates, that are used in the calculation of $p_{\mathrm{T}}^{\text {miss }}$, are considered and found to be negligible. 
The uncertainties in the normalization of the single top quark and V+jets background sources are based on measurements performed in [56-58] and take into account an extrapolation to the current analysis phase space. They are estimated to be $\pm 30 \%$ and $\pm 50 \%$ respectively and typically result in a normalization uncertainty that is negligible. The uncertainty in the normalization and shape of the multijet QCD background is estimated by using alternative control regions containing conversion electrons in the e+jets channel and muons with $I_{\text {rel }}>0.3$ in the $\mu+$ jets channel. This effectively varies the total normalization of the multijet QCD background by up to $60 \%$, and also the shape of the contribution by up to $\pm 30 \%$ in any one bin, but is found to result in a negligible uncertainty after unfolding, except at large $\left|\eta^{\ell}\right|$.

Uncertainties in the top quark mass are estimated by using simulated $t \bar{t}$ samples where the top quark mass has been varied up and down by $1 \mathrm{GeV}$, which is comparable to the uncertainty in the measured top quark mass [59].

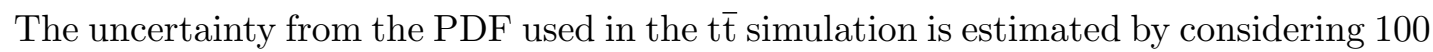
independent replicas of NNPDF30_nlo_as_0118. The RMS of the uncertainties originating from the variation of each replica is taken as the PDF uncertainty. The uncertainty resulting from using the NNPDF30_nlo_as_0118 set derived with varied values of $\alpha_{S}$ is combined in quadrature with the PDF uncertainty.

The uncertainty arising from the mismodeling of the top quark $p_{\mathrm{T}}$ spectrum is estimated by reweighting the $p_{\mathrm{T}}$ distribution in simulation to match that measured by the previous measurements $[7,8]$. The reweighting varies the yield of simulated t $\bar{t}$ events in the bins of the measurement by up to $20 \%$, and results in a negligible uncertainty in the measured cross section.

Several sources of uncertainty for the modeling of the parton shower in the simulated POWHEG+PYTHIA sample are considered.

The uncertainty originating from the parton shower scale used when simulating the initial-state radiation is estimated by varying the scale up and down by a factor of two. Similarly the uncertainty originating from the scale for final-state radiation, which is constrained by measurements made at the LEP collider [60], is estimated by varying the scale up and down by a factor of $\sqrt{2}$. The renormalization and factorization scales used in the matrix-element calculations are also varied independently by factors of 0.5 and 2 . An additional variation is performed where both scales are varied simultaneously by the same factors. The shower scale uncertainty is defined as the envelope of the parton shower scale uncertainties and the matrix-element scale uncertainties.

The systematic uncertainty in matching the matrix-element to the parton shower is determined by varying the parameter $h_{\text {damp }}$, which regulates the high- $p_{\mathrm{T}}$ radiation by damping real emission generated in POWHEG, within its uncertainties. The parameter is set to $h_{\text {damp }}=1.58_{-0.59}^{+0.66}$ multiplied by the mass of the top quark in the CUETP8M2T4 tune [19]. The parameters controlling the underlying event in the CUETP8M2T4 tune are also varied to estimate the uncertainty in this source [19].

The uncertainty in the modeling of the momentum transfer from $b$ quarks to $b$ hadrons is estimated by reweighting the tuned quantity $x_{\mathrm{b}}=p_{\mathrm{T}}(\mathrm{B}) / p_{\mathrm{T}}(\mathrm{b}$ jet $)$ for each particle-level b-tagged jet within its uncertainties, where $p_{\mathrm{T}}(\mathrm{B})$ is the transverse momentum of the $\mathrm{b}$ 
hadron, and $p_{\mathrm{T}}$ (b jet) is the transverse momentum of the particle-level b-tagged jet. The difference when using an alternative model (the Peterson model [61]) for the fragmentation of $\mathrm{b}$ quarks is also included as an additional uncertainty. The energy response of b jets is sensitive to the single-lepton branching fractions of $\mathrm{b}$ hadrons, and the uncertainty originating from the choice of branching fractions in the POWHEG+PYTHIA simulation is estimated by reweighting the branching fractions to those reported in ref. [59].

The effects of any mismodeling of the color reconnection in the simulation are estimated by comparing the cross sections obtained with samples including and excluding the effects of color reconnection on the decay products of the top quarks (Early resonance decays). A comparison to two samples obtained with alternative models of color reconnection are also included, one where QCD color rules are considered in the simulation of the color reconnection (QCD-based) [62], and another where gluons can be moved to different color strings during the simulation of the color reconnection (Gluon move) [63].

The statistical uncertainty arising from the finite size of the POWHEG+PYTHIA sample, which is used to construct the nominal response matrix, is propagated to the final measurement. This uncertainty is negligible.

Each source of systematic uncertainty is summarised for each variable in table 1, where the minimum and maximum relative uncertainty in the normalized differential cross section (over all bins) are shown. The minimum and maximum of the total relative uncertainty over all bins are also shown. Sources of uncertainties in the calculation of $p_{\mathrm{T}}^{\text {miss }}$ do not affect some distributions, and are indicated in the table by - . The dominant uncertainty in the measurement of the normalized cross sections comes from the uncertainty in the JES. Other significant uncertainties come from the theoretical modeling of t $\bar{t}$ production in simulation, in particular from the uncertainty in the shower scale for final-state radiation. A similar table for the absolute differential cross section uncertainties is shown in appendix C. The uncertainty in the JES is also significant in the measurements of the absolute cross sections, however the uncertainty in the final-state radiation scale becomes dominant. The total uncertainty from all sources in the normalized cross section is typically below $5 \%$ in each bin, and can be as large as $21 \%$. For the measurements of the absolute cross section, the total uncertainty is typically $10 \%$, and can be as large as $22 \%$. 


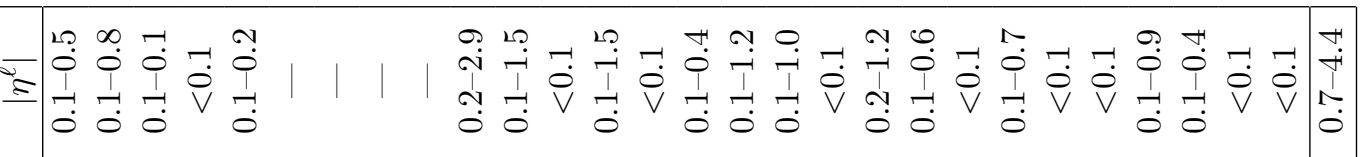

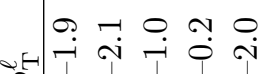

(1)

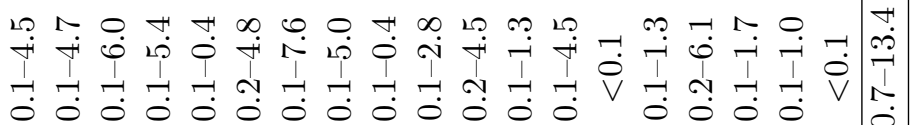

Ґ サ

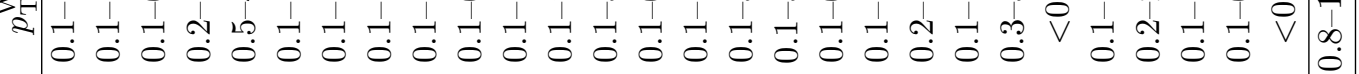

急

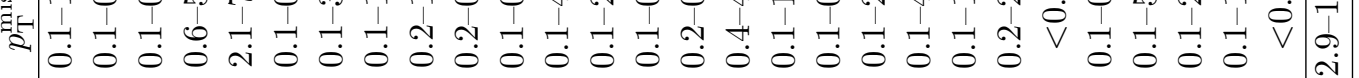

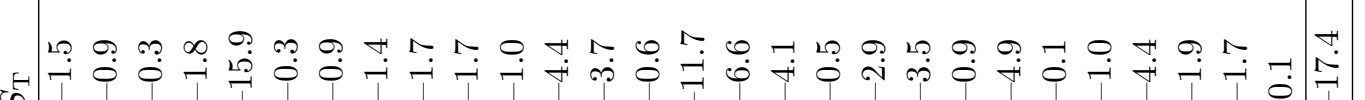

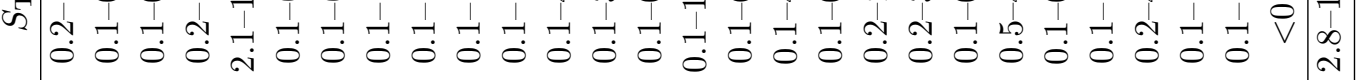

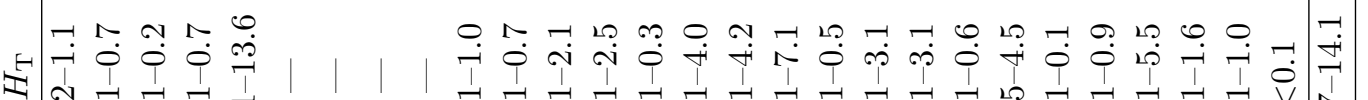

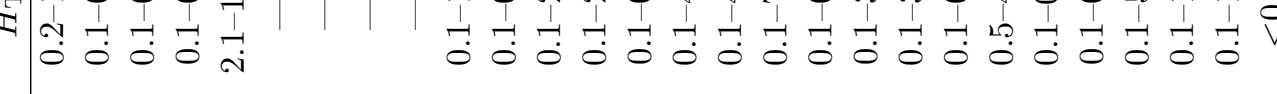

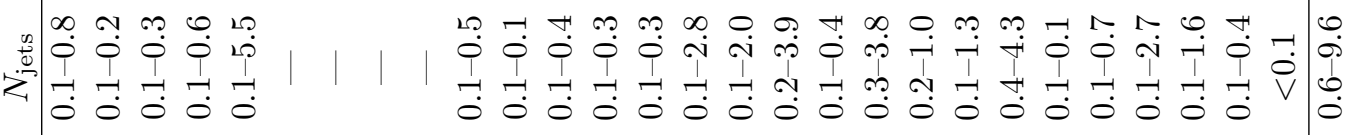

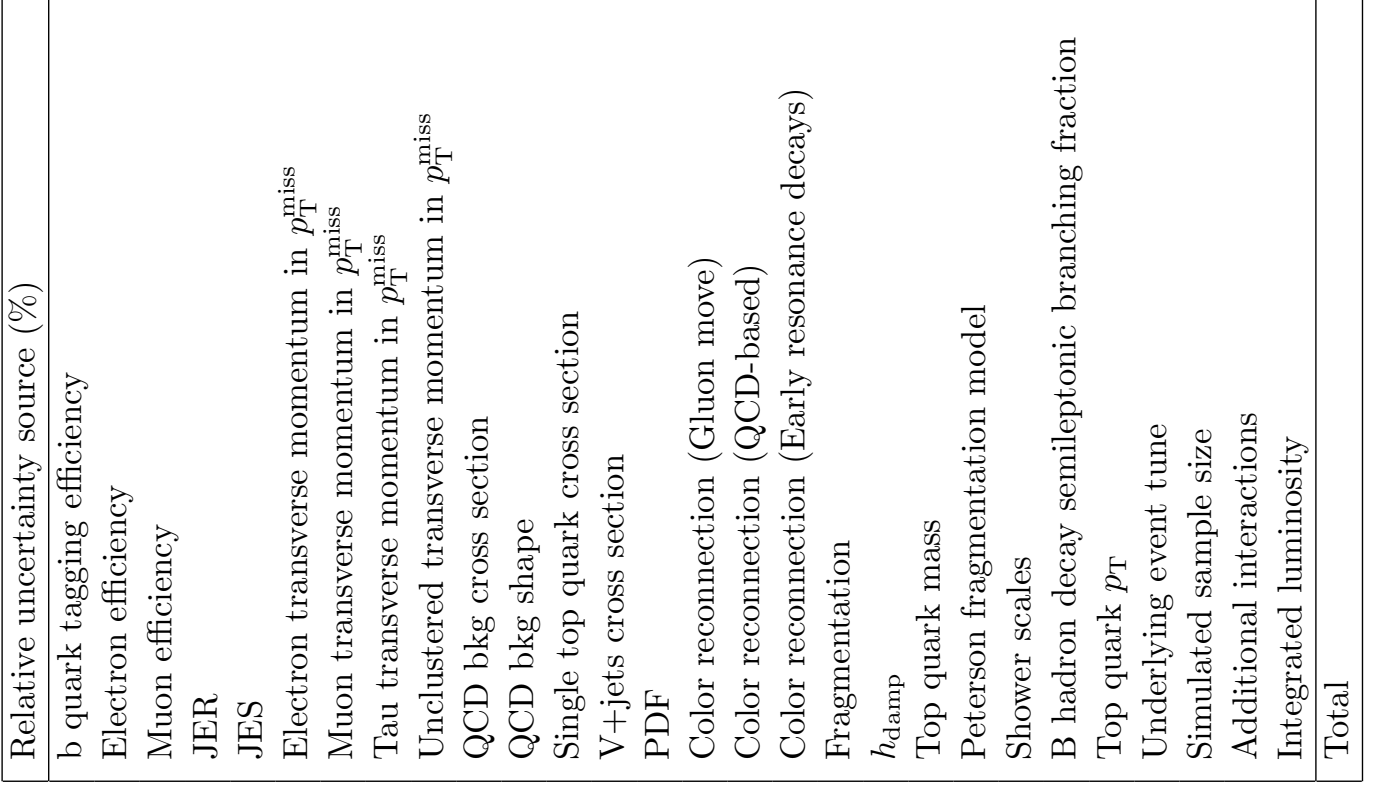




\section{Cross section results}

The normalized differential t $\overline{\mathrm{t}}$ production cross section with respect to $N_{\text {jets }}$ is shown in figure 3 , with respect to $H_{\mathrm{T}}$ and $S_{\mathrm{T}}$ in figure 4 , with respect to $p_{\mathrm{T}}^{\text {miss }}$ and $p_{\mathrm{T}}^{\mathrm{W}}$ in figure 5 and with respect to $p_{\mathrm{T}}^{\ell}$ and $\left|\eta^{\ell}\right|$ in figure 6 . Tabulated results are listed in appendix A. Measurements of the absolute differential t $\overline{\mathrm{t}}$ production cross sections are shown in figures $7,8,9$ and 10, and tabulated in appendix B. In each figure, the measured cross section is compared with the predictions from several combinations of matrix-element and parton shower generators, namely POWHEG+PYTHIA, POWHEG+HERWIG++, MG5_aMC@NLO-NLO, and MG5_aMC@NLO-LO. Each measured cross section is also compared to the POWHEG+PYTHIA generator after varying the shower scales and the $h_{\text {damp }}$ parameter used in generating the sample within their uncertainties, and also after reweighting the top quark $p_{\mathrm{T}}$ as described in section 6 .

The level of agreement between the measured and predicted differential cross sections are determined through a $\chi^{2}$ test, where the full covariance matrix, including the correlations between the statistical and systematic uncertainties in each bin of the measurements, is taken into account. The results, including the $p$-value of each test, are shown in tables 2 and 3 .

The predictions of the POWHEG+PYTHIA model are consistent with data for the $N_{\text {jets }}$, $p_{\mathrm{T}}^{\text {miss }}, S_{\mathrm{T}}$, and $p_{\mathrm{T}}^{\ell}$ distributions. In particular, the prediction of the $N_{\text {jets }}$ distribution has a $\chi^{2}$ per degree of freedom of $2 / 5$ for the normalized and 2.2/6 for the absolute cross section measurement. The jet multiplicity from previous $8 \mathrm{TeV}$ measurements was used in deriving the CUETP8M2T4 tune [19], and this confirms that the tune continues to accurately describe the jet multiplicity on a larger data set with a higher $\sqrt{s}$. On the other hand, tensions are observed for the $H_{\mathrm{T}}, p_{\mathrm{T}}^{\mathrm{W}}$ and $\left|\eta^{\ell}\right|$ variables. An additional $\chi^{2}$ calculation between the POWHEG+PYTHIA model and unfolded data is performed, where the theoretical uncertainties within the generator, described in section 6 , are included, as well as in the unfolded data. The correlations between the uncertainties in the prediction of the generator and the unfolded data are taken into account. The result of this test demonstrates that the theoretical uncertainties in the POWHEG+PYTHIA model cover the differences between the POWHEG+PYTHIA model and the unfolded data in the phase space analyzed.

The POWHEG+HERWIG++ and MG5_aMC@NLO-NLO models are broadly consistent with the unfolded data, even without including the theoretical uncertainties in the $\chi^{2}$ test, with the exception of $N_{\text {jets }}$ in POWHEG+HERWIG++ and $\left|\eta^{\ell}\right|$ in MG5_aMC@NLO-NLO. Without these uncertainties, the MG5_aMC@NLO-LO model is not compatible with any kinematic event distribution in the unfolded data presented here.

The effect of the regularization in the unfolding procedure is investigated by unfolding without regularization, which typically results in a small change in the $\chi^{2}$. When unfolding without regularization, the largest changes in $\chi^{2}$ for the normalized cross sections are for the $H_{\mathrm{T}}$ distribution with the MG5_amC@NLO-NLO model, where the $\chi^{2}$ per degree of freedom increases from $11 / 12$ to $12 / 12$, and for the $p_{\mathrm{T}}^{\text {miss }}$ distribution in the POWHEG+PYTHIA model (including the model theoretical uncertainties), where the $\chi^{2}$ per degree of freedom 


\begin{tabular}{|ccccc|}
\hline & \multicolumn{2}{c|}{ POWHEG+PYTHIA } & \multicolumn{2}{l|}{ With MC theoretical uncertainties } \\
& $\chi^{2} /$ ndf & $p$-value & $\chi^{2} /$ ndf & $p$-value \\
\hline$N_{\text {jets }}$ & $2 / 5$ & 0.85 & $1.5 / 5$ & 0.91 \\
$H_{\mathrm{T}}$ & $26 / 12$ & $<0.01$ & $4.8 / 12$ & 0.97 \\
$S_{\mathrm{T}}$ & $22 / 12$ & 0.04 & $4.2 / 12$ & 0.98 \\
$p_{\mathrm{T}}^{\mathrm{miss}}$ & $11 / 5$ & 0.06 & $2.9 / 5$ & 0.72 \\
$p_{\mathrm{T}}^{\mathrm{W}}$ & $16 / 6$ & 0.01 & $2.5 / 6$ & 0.87 \\
$p_{\mathrm{T}}^{\ell}$ & $24 / 16$ & 0.09 & $14 / 16$ & 0.63 \\
$\left|\eta^{\ell}\right|$ & $19 / 7$ & $<0.01$ & $15 / 7$ & 0.04 \\
\hline
\end{tabular}

\begin{tabular}{|ccccccc|}
\hline \multicolumn{5}{|c}{ POwheg+Herwig ++} & MG5_amC@NLO-NLO+PYTHIA & \multicolumn{2}{c|}{ MG5_amC@NLO-LO+PYTHIA } \\
& $\chi^{2} /$ ndf & $p$-value & $\chi^{2} /$ ndf & $p$-value & $\chi^{2} /$ ndf & $p$-value \\
\hline$N_{\text {jets }}$ & $38 / 5$ & $<0.01$ & $9.5 / 5$ & 0.09 & $78 / 5$ & $<0.01$ \\
$H_{\mathrm{T}}$ & $23 / 12$ & 0.03 & $11 / 12$ & 0.52 & $160 / 12$ & $<0.01$ \\
$S_{\mathrm{T}}$ & $21 / 12$ & 0.04 & $11 / 12$ & 0.57 & $110 / 12$ & $<0.01$ \\
$p_{\mathrm{T}}^{\mathrm{miss}}$ & $1.3 / 5$ & 0.93 & $5.9 / 5$ & 0.31 & $23 / 5$ & $<0.01$ \\
$p_{\mathrm{T}}^{\mathrm{W}}$ & $0.81 / 6$ & 0.99 & $8.9 / 6$ & 0.18 & $30 / 6$ & $<0.01$ \\
$p_{\mathrm{T}}^{\ell}$ & $11 / 16$ & 0.82 & $16 / 16$ & 0.44 & $37 / 16$ & $<0.01$ \\
$\left|\eta^{\ell}\right|$ & $19 / 7$ & $<0.01$ & $24 / 7$ & $<0.01$ & $30 / 7$ & $<0.01$ \\
\hline
\end{tabular}

Table 2. Results of a goodness-of-fit test between the normalized cross sections in data and several models, with values given as $\chi^{2} /$ number of degrees of freedom (ndf).

decreases from $2.9 / 5$ to $2.1 / 5$. The effects on the $\chi^{2}$ for all other variables and models are small. The $\chi^{2}$ does not change for the $p_{\mathrm{T}}^{\ell}$ and $\left|\eta^{\ell}\right|$ distributions for any model when unfolding without regularization. 


\begin{tabular}{|ccccc|}
\hline & \multicolumn{2}{c|}{ POWHEG+PYTHIA } & \multicolumn{2}{c|}{ With MC theoretical uncertainties } \\
& $\chi^{2} /$ ndf & $p$-value & $\chi^{2} /$ ndf & $p$-value \\
\hline$N_{\text {jets }}$ & $2.2 / 6$ & 0.90 & $1.7 / 6$ & 0.95 \\
$H_{\mathrm{T}}$ & $23 / 13$ & 0.05 & $4.3 / 13$ & 0.99 \\
$S_{\mathrm{T}}$ & $19 / 13$ & 0.11 & $4.7 / 13$ & 0.98 \\
$p_{\mathrm{T}}^{\mathrm{miss}}$ & $13 / 6$ & 0.05 & $3.1 / 6$ & 0.80 \\
$p_{\mathrm{T}}^{\mathrm{W}}$ & $17 / 7$ & 0.02 & $2.7 / 7$ & 0.91 \\
$p_{\mathrm{T}}^{\ell}$ & $20 / 17$ & 0.28 & $14 / 17$ & 0.68 \\
$\left|\eta^{\ell}\right|$ & $16 / 8$ & 0.04 & $15 / 8$ & 0.06 \\
\hline
\end{tabular}

\begin{tabular}{|ccccccc|}
\hline \multicolumn{5}{|c}{ POWHEG+HERWIG++ } & MG5_amC@NLO-NLO+PYTHIA & \multicolumn{2}{c|}{ MG5_aMC@NLO-LO+PYTHIA } \\
& $\chi^{2} /$ ndf & $p$-value & $\chi^{2} /$ ndf & $p$-value & $\chi^{2} /$ ndf & $p$-value \\
\hline$N_{\text {jets }}$ & $39 / 6$ & $<0.01$ & $12 / 6$ & 0.07 & $93 / 6$ & $<0.01$ \\
$H_{\mathrm{T}}$ & $21 / 13$ & 0.07 & $10 / 13$ & 0.66 & $150 / 13$ & $<0.01$ \\
$S_{\mathrm{T}}$ & $18 / 13$ & 0.17 & $9.3 / 13$ & 0.75 & $110 / 13$ & $<0.01$ \\
$p_{\mathrm{T}}^{\mathrm{miss}}$ & $1.5 / 6$ & 0.96 & $6.6 / 6$ & 0.36 & $26 / 6$ & $<0.01$ \\
$p_{\mathrm{T}}^{\mathrm{W}}$ & $0.90 / 7$ & 1.00 & $9.2 / 7$ & 0.24 & $33 / 7$ & $<0.01$ \\
$p_{\mathrm{T}}^{\ell}$ & $11 / 17$ & 0.87 & $15 / 17$ & 0.58 & $36 / 17$ & $<0.01$ \\
$\left|\eta^{\ell}\right|$ & $17 / 8$ & 0.04 & $23 / 8$ & $<0.01$ & $31 / 8$ & $<0.01$ \\
\hline
\end{tabular}

Table 3. Results of a goodness-of-fit test between the absolute cross sections in data and several models, with values given as $\chi^{2} /$ number of degrees of freedom (ndf). 

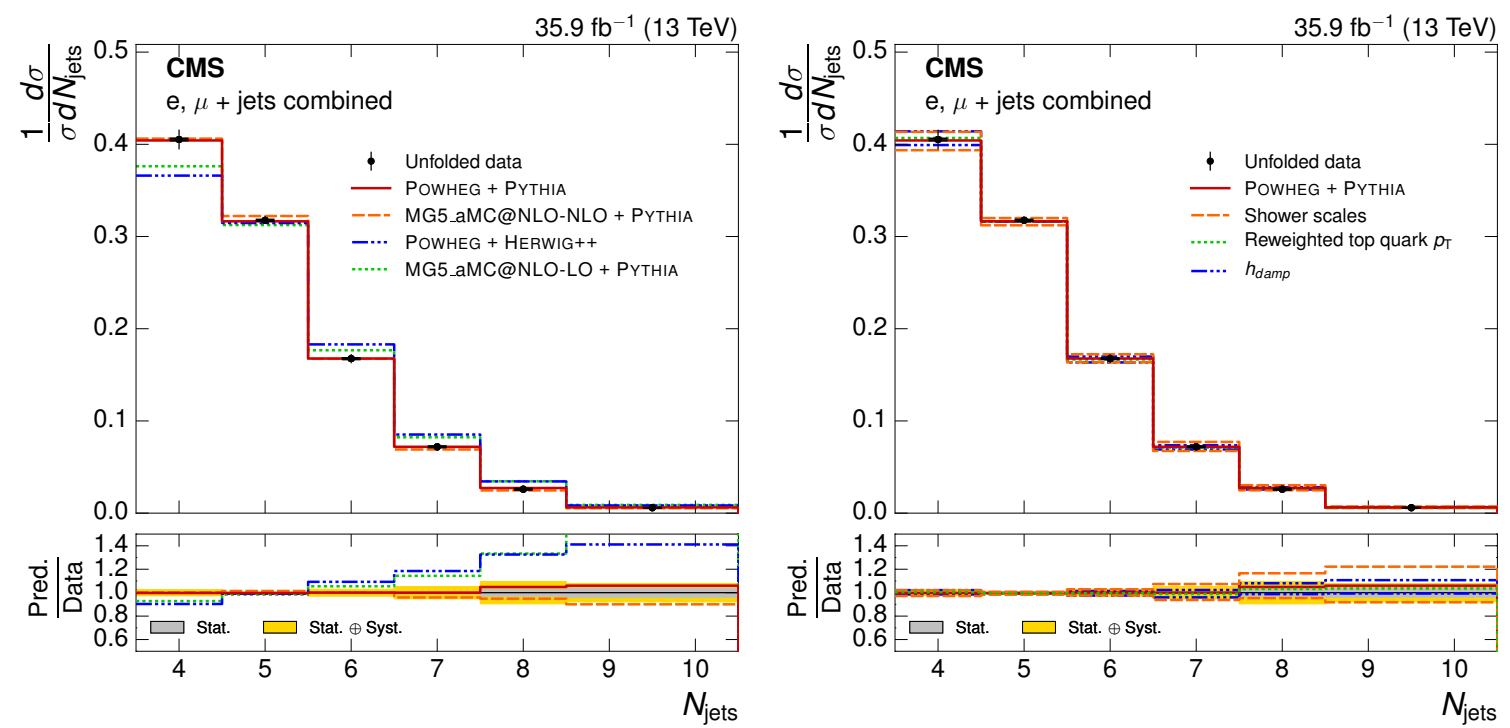

Figure 3. Normalized $N_{\text {jets }}$ differential t $\overline{\mathrm{t}}$ cross section, compared to different $\mathrm{t} \overline{\mathrm{t}}$ simulations in the left plot, and compared to the POWHEG+PYTHIA simulation after varying the shower scales, and $h_{\text {damp }}$ parameter, within their uncertainties, in the right plot. The vertical bars on the data represent the statistical and systematic uncertainties added in quadrature. The bottom panels show the ratio of the predictions to the data. 

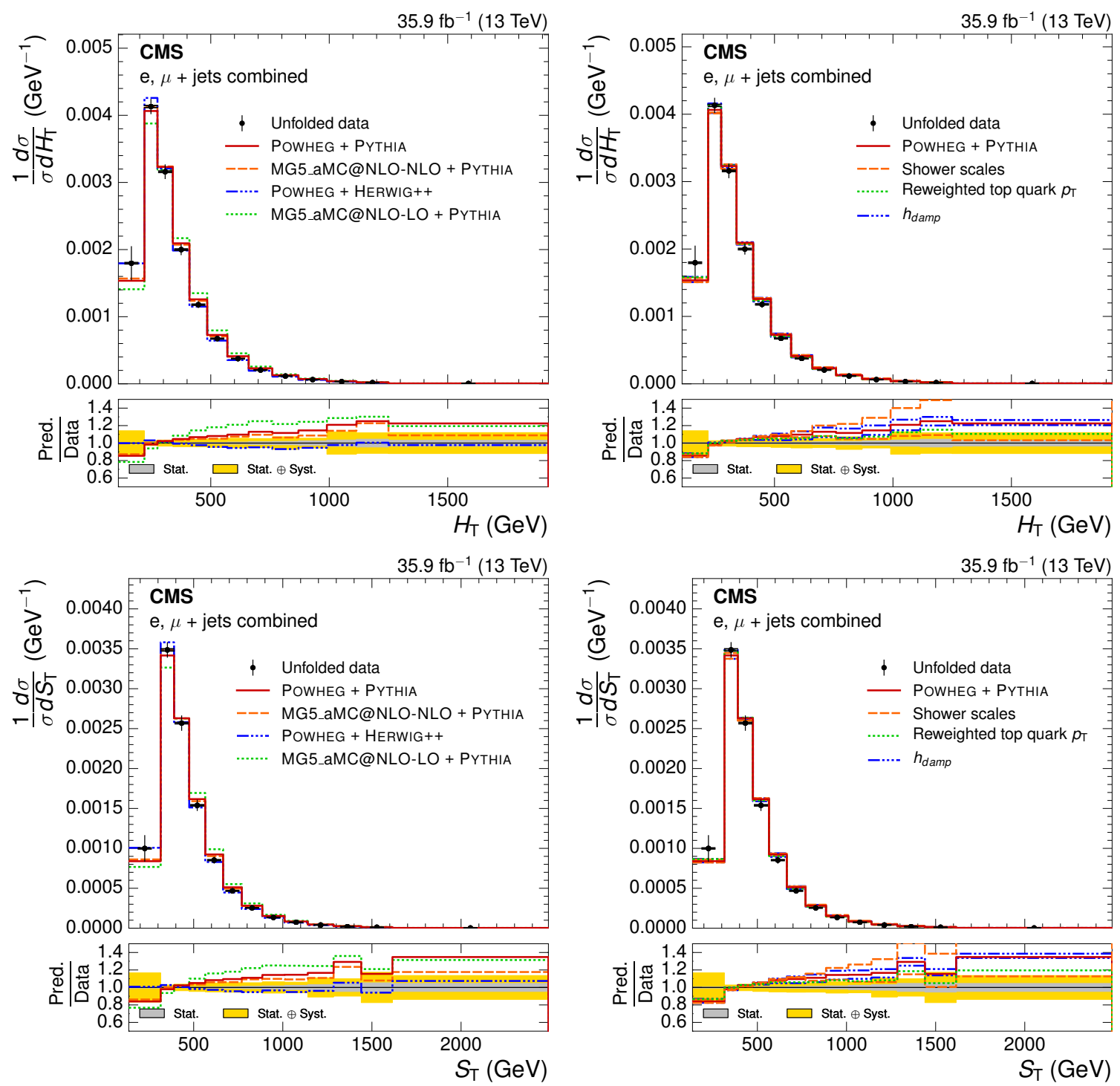

Figure 4. Normalized $H_{\mathrm{T}}$ (upper) and $S_{\mathrm{T}}$ (lower) differential t $\overline{\mathrm{t}}$ cross sections, compared to different $t \bar{t}$ simulations in the left plots, and compared to the POWHEG+PYTHIA simulation after varying the shower scales, and $h_{\text {damp }}$ parameter, within their uncertainties, in the right plots. The vertical bars on the data represent the statistical and systematic uncertainties added in quadrature. The bottom panels show the ratio of the predictions to the data. 

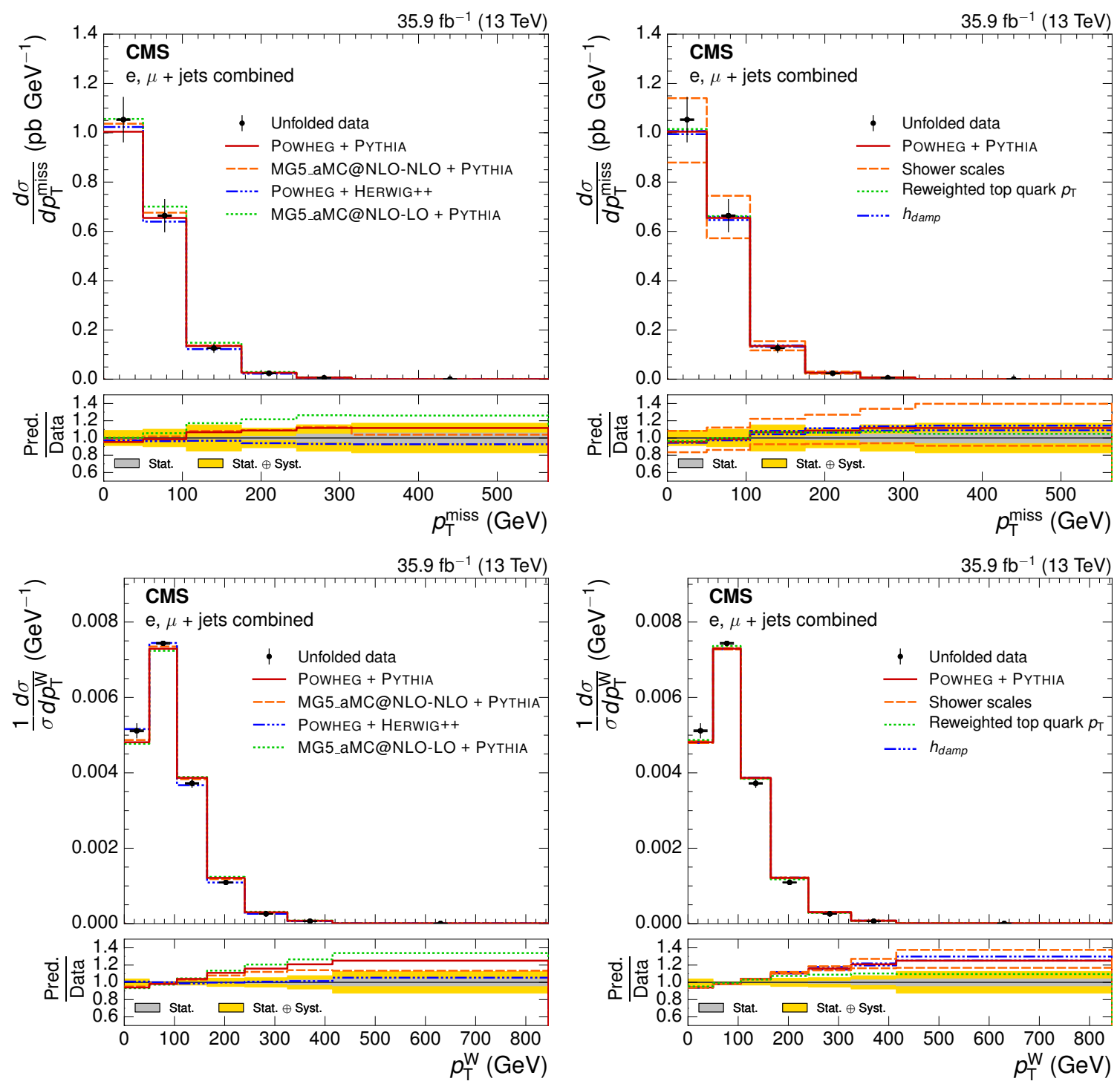

Figure 5. Normalized $p_{\mathrm{T}}^{\text {miss }}$ (upper) and $p_{\mathrm{T}}^{\mathrm{W}}$ (lower) differential t $\overline{\mathrm{t}}$ cross sections, compared to different $\mathrm{t} \overline{\mathrm{t}}$ simulations in the left plots, and compared to the POWHEG+PYTHIA simulation after varying the shower scales, and $h_{\text {damp }}$ parameter, within their uncertainties, in the right plots. The vertical bars on the data represent the statistical and systematic uncertainties added in quadrature. The bottom panels show the ratio of the predictions to the data. 

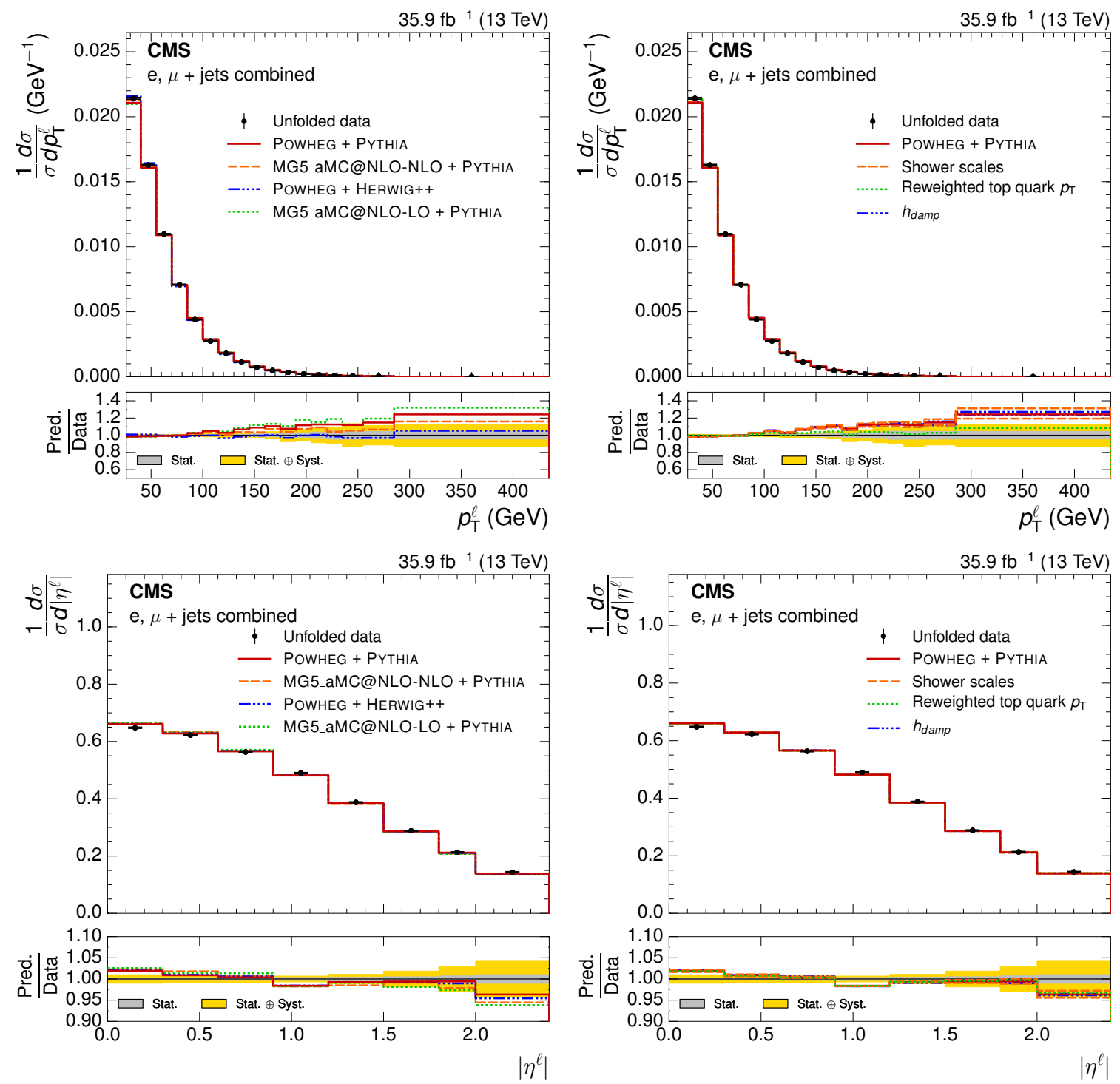

Figure 6. Normalized $p_{\mathrm{T}}^{\ell}$ (upper) and $\left|\eta^{\ell}\right|$ (lower) differential t $\overline{\mathrm{t}}$ cross sections, compared to different $\mathrm{t} \overline{\mathrm{t}}$ simulations in the left plots, and compared to the POWHEG+PYTHIA simulation after varying the shower scales, and $h_{\text {damp }}$ parameter, within their uncertainties, in the right plots. The vertical bars on the data represent the statistical and systematic uncertainties added in quadrature. The bottom panels show the ratio of the predictions to the data. 

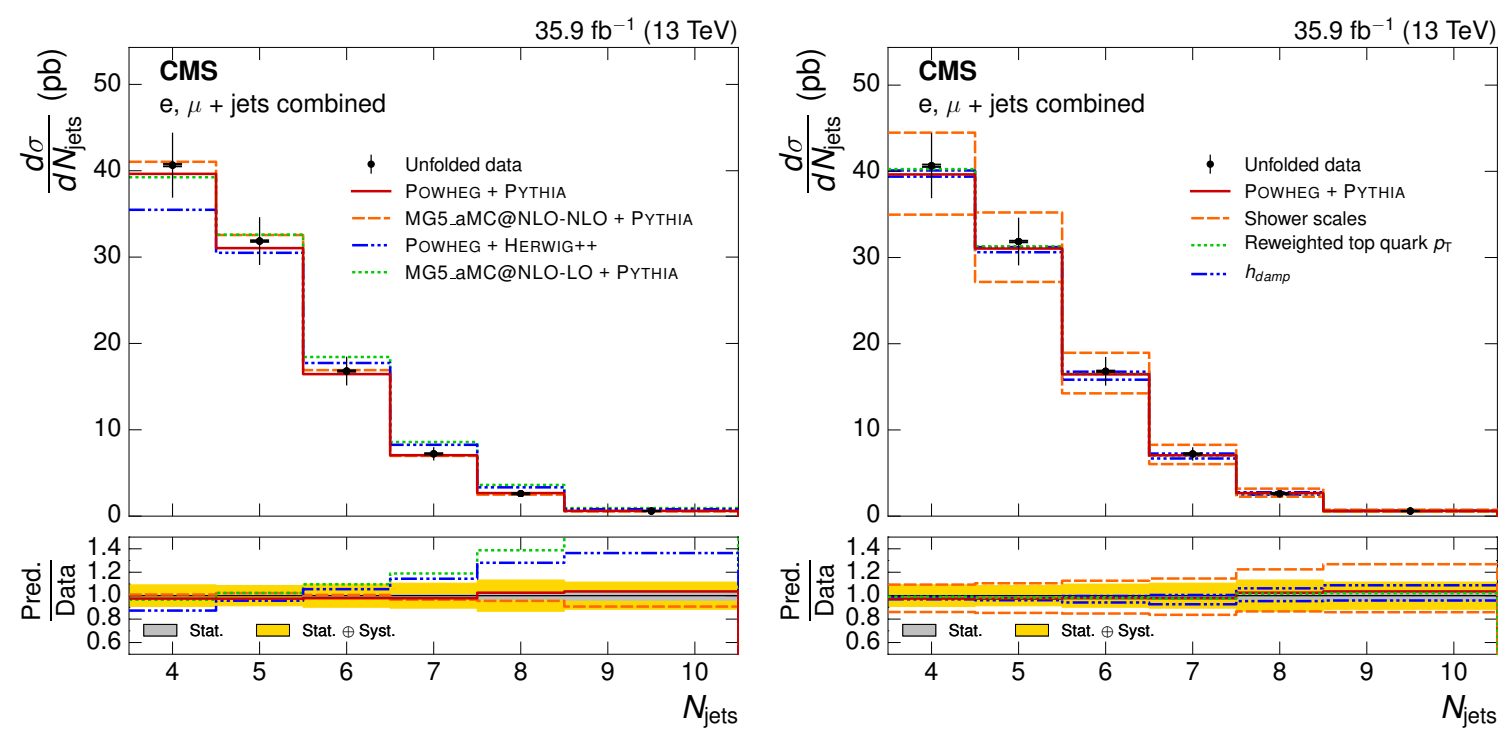

Figure 7. Absolute $N_{\text {jets }}$ differential $t \bar{t}$ cross section, compared to different $t \bar{t}$ simulations in the left plot, and compared to the POWHEG+PYTHIA simulation after varying the shower scales, and $h_{\text {damp }}$ parameter, within their uncertainties, in the right plot. The vertical bars on the data represent the statistical and systematic uncertainties added in quadrature. The bottom panels show the ratio of the predictions to the data. 

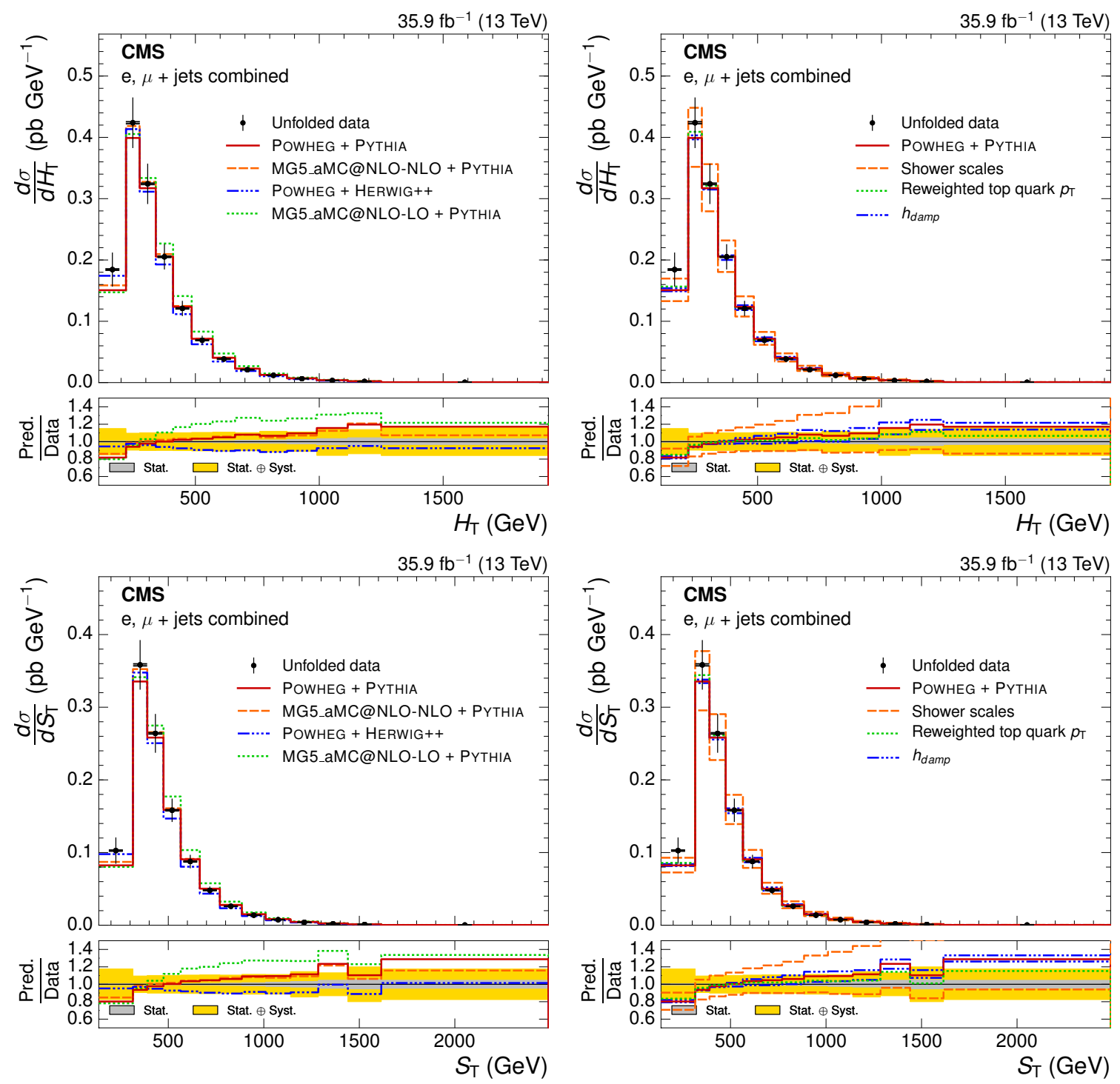

Figure 8. Absolute $H_{\mathrm{T}}$ (upper) and $S_{\mathrm{T}}$ (lower) differential t $\overline{\mathrm{t}}$ cross sections, compared to different $\mathrm{t} \overline{\mathrm{t}}$ simulations in the left plots, and compared to the POWHEG+PYTHIA simulation after varying the shower scales, and $h_{\text {damp }}$ parameter, within their uncertainties, in the right plots. The vertical bars on the data represent the statistical and systematic uncertainties added in quadrature. The bottom panels show the ratio of the predictions to the data. 

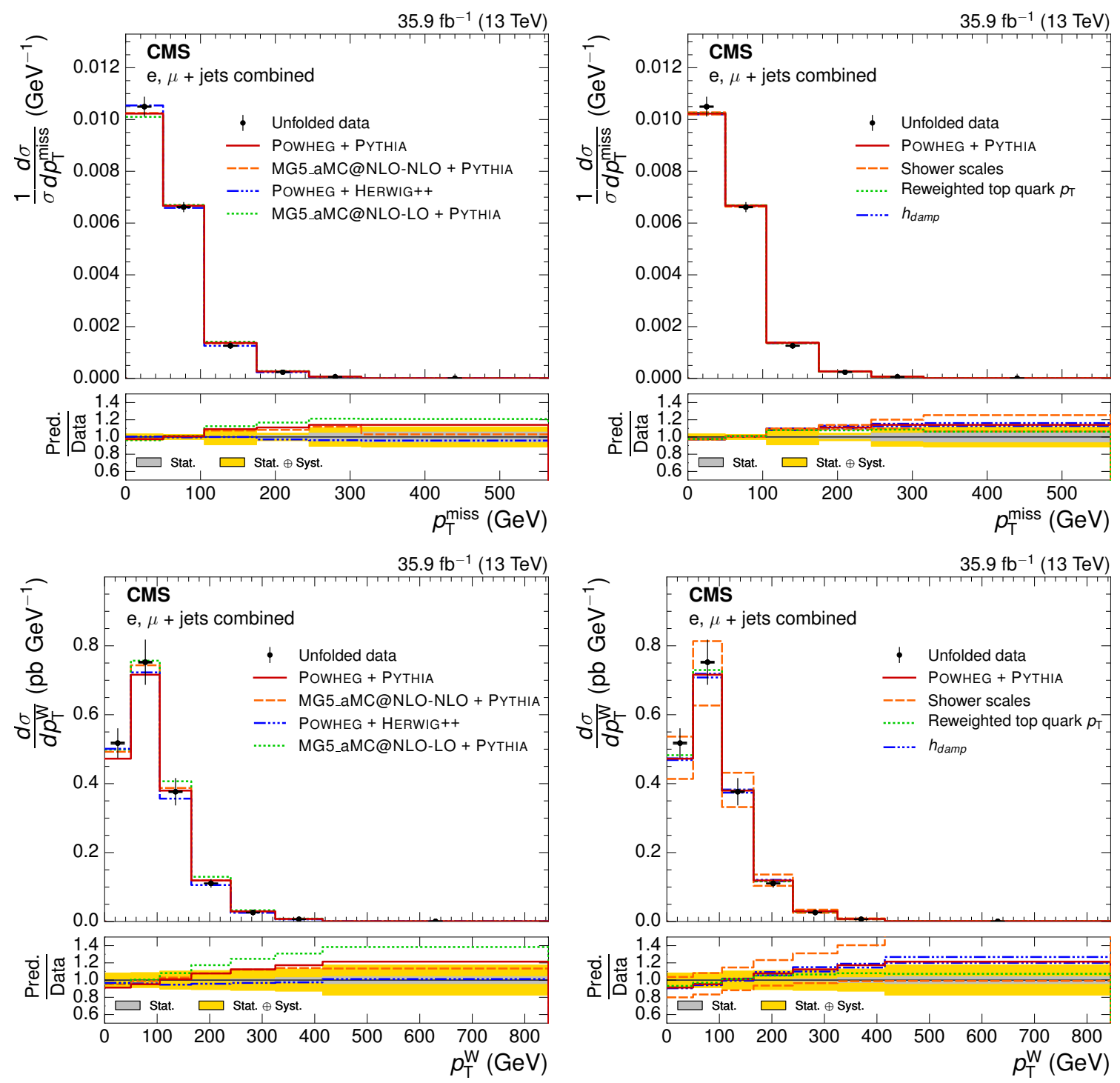

Figure 9. Absolute $p_{\mathrm{T}}^{\text {miss }}$ (upper) and $p_{\mathrm{T}}^{\mathrm{W}}$ (lower) differential t $\overline{\mathrm{t}}$ cross sections, compared to different $\mathrm{t} \overline{\mathrm{t}}$ simulations in the left plots, and compared to the POWHEG+PYTHIA simulation after varying the shower scales, and $h_{\text {damp }}$ parameter, within their uncertainties, in the right plots. The vertical bars on the data represent the statistical and systematic uncertainties added in quadrature. The bottom panels show the ratio of the predictions to the data. 

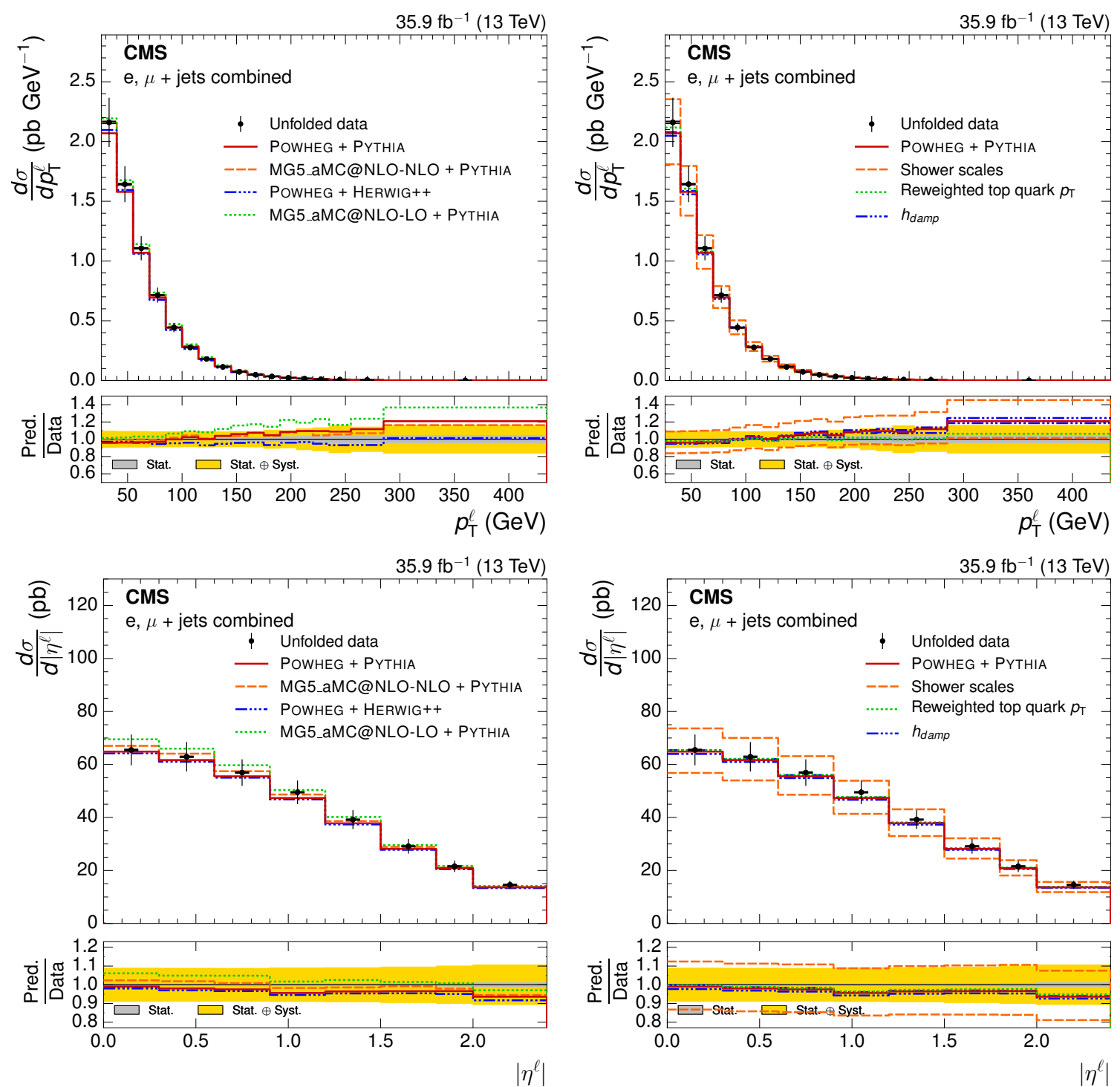

Figure 10. Absolute $p_{\mathrm{T}}^{\ell}$ (upper) and $\left|\eta^{\ell}\right|$ (lower) differential t $\overline{\mathrm{t}}$ cross sections, compared to different $\mathrm{t} \overline{\mathrm{t}}$ simulations in the left plots, and compared to the POWHEG+PYTHIA simulation after varying the shower scales, and $h_{\text {damp }}$ parameter, within their uncertainties, in the right plots. The vertical bars on the data represent the statistical and systematic uncertainties added in quadrature. The bottom panels show the ratio of the predictions to the data. 


\section{Summary}

Normalized and absolute differential t $\bar{t}$ production cross sections with respect to several kinematic event variables are measured at the particle level in a visible phase space region. The results are based on proton-proton collision data at $\sqrt{s}=13 \mathrm{TeV}$, collected by the CMS experiment with an integrated luminosity of $35.9 \mathrm{fb}^{-1}$. The total cross section is observed to be consistent with previous results and next-to-next-to-leading-order calculations, and the differential measurements are compared to several t $\overline{\mathrm{t}}$ production models: POWHEG+PYTHIA, POWHEG+HERWIG++, MG5_aMC@NLO-LO, and MG5_aMC@NLO-NLO.

The POWHEG+PYTHIA simulation is found to be generally consistent with the data, with residual differences covered by theoretical uncertainties. The jet multiplicity distribution is particularly well-modeled, having been tuned on $\mathrm{LHC} 8 \mathrm{TeV}$ data. The POWHEG+HERWIG++ and MG5_aMC@NLO-NLO models are shown to be consistent with data for most kinematic event variables, while the MG5_aMC@NLO-LO model does not provide an accurate description of any variable measured in the data.

It is expected that the results presented here will be useful for tuning t $\overline{\mathrm{t}}$ generators and models in the future. To facilitate this, the measurements presented here have been implemented in the RIVET framework and will be available to the wider community.

\section{Acknowledgments}

We congratulate our colleagues in the CERN accelerator departments for the excellent performance of the LHC and thank the technical and administrative staffs at CERN and at other CMS institutes for their contributions to the success of the CMS effort. In addition, we gratefully acknowledge the computing centers and personnel of the Worldwide LHC Computing Grid for delivering so effectively the computing infrastructure essential to our analyses. Finally, we acknowledge the enduring support for the construction and operation of the LHC and the CMS detector provided by the following funding agencies: BMWFW and FWF (Austria); FNRS and FWO (Belgium); CNPq, CAPES, FAPERJ, and FAPESP (Brazil); MES (Bulgaria); CERN; CAS, MoST, and NSFC (China); COLCIENCIAS (Colombia); MSES and CSF (Croatia); RPF (Cyprus); SENESCYT (Ecuador); MoER, ERC IUT, and ERDF (Estonia); Academy of Finland, MEC, and HIP (Finland); CEA and CNRS/IN2P3 (France); BMBF, DFG, and HGF (Germany); GSRT (Greece); OTKA and NIH (Hungary); DAE and DST (India); IPM (Iran); SFI (Ireland); INFN (Italy); MSIP and NRF (Republic of Korea); LAS (Lithuania); MOE and UM (Malaysia); BUAP, CINVESTAV, CONACYT, LNS, SEP, and UASLP-FAI (Mexico); MBIE (New Zealand); PAEC (Pakistan); MSHE and NSC (Poland); FCT (Portugal); JINR (Dubna); MON, RosAtom, RAS, RFBR and RAEP (Russia); MESTD (Serbia); SEIDI, CPAN, PCTI and FEDER (Spain); Swiss Funding Agencies (Switzerland); MST (Taipei); ThEPCenter, IPST, STAR, and NSTDA (Thailand); TUBITAK and TAEK (Turkey); NASU and SFFR (Ukraine); STFC (United Kingdom); DOE and NSF (U.S.A.).

Individuals have received support from the Marie-Curie program and the European Research Council and Horizon 2020 Grant, contract No. 675440 (European Union); 
the Leventis Foundation; the A. P. Sloan Foundation; the Alexander von Humboldt Foundation; the Belgian Federal Science Policy Office; the Fonds pour la Formation à la Recherche dans l'Industrie et dans l'Agriculture (FRIA-Belgium); the Agentschap voor Innovatie door Wetenschap en Technologie (IWT-Belgium); the F.R.S.-FNRS and FWO (Belgium) under the "Excellence of Science - EOS" - be.h project n. 30820817; the Ministry of Education, Youth and Sports (MEYS) of the Czech Republic; the Council of Science and Industrial Research, India; the HOMING PLUS program of the Foundation for Polish Science, cofinanced from European Union, Regional Development Fund, the Mobility Plus program of the Ministry of Science and Higher Education, the National Science Center (Poland), contracts Harmonia 2014/14/M/ST2/00428, Opus 2014/13/B/ST2/02543, 2014/15/B/ST2/03998, and 2015/19/B/ST2/02861, Sonata-bis 2012/07/E/ST2/01406; the National Priorities Research Program by Qatar National Research Fund; the Programa Severo Ochoa del Principado de Asturias; the Thalis and Aristeia programs cofinanced by EU-ESF and the Greek NSRF; the Rachadapisek Sompot Fund for Postdoctoral Fellowship, Chulalongkorn University and the Chulalongkorn Academic into Its 2nd Century Project Advancement Project (Thailand); the Welch Foundation, contract C-1845; and the Weston Havens Foundation (U.S.A.).

\section{A Tabulated normalized differential t $\bar{t}$ production cross sections}

\begin{tabular}{|cccc|}
\hline$N_{\text {jets }}$ & $\frac{1}{\mathrm{~d} \sigma} \frac{\mathrm{d} \sigma}{\mathrm{d} N_{\text {jets }}}$ & $\begin{array}{c}\text { Stat. unc. } \\
(\%)\end{array}$ & $\begin{array}{c}\text { Syst. unc. } \\
(\%)\end{array}$ \\
\hline $3.5-4.5$ & 0.405 & 0.27 & 2.6 \\
$4.5-5.5$ & 0.318 & 0.27 & 0.65 \\
$5.5-6.5$ & 0.168 & 0.47 & 3.0 \\
$6.5-7.5$ & $7.19 \times 10^{-2}$ & 1.0 & 5.3 \\
$7.5-8.5$ & $2.60 \times 10^{-2}$ & 3.0 & 9.6 \\
$8.5-10.5$ & $5.89 \times 10^{-3}$ & 3.9 & 7.8 \\
\hline
\end{tabular}

Table 4. Results of the normalised differential cross sections with relative uncertainties in the combined channel with respect to $N_{\text {jets }}$. 


\begin{tabular}{|cccc|}
\hline $\begin{array}{c}H_{\mathrm{T}} \\
(\mathrm{GeV})\end{array}$ & $\begin{array}{c}\frac{1}{\mathrm{~d} \sigma} \frac{\mathrm{d} \sigma}{\mathrm{d} H_{\mathrm{T}}} \\
\left(\mathrm{GeV}^{-1}\right)\end{array}$ & $\begin{array}{c}\text { Stat. unc. } \\
(\%)\end{array}$ & $\begin{array}{c}\text { Syst. unc. } \\
(\%)\end{array}$ \\
\hline $110-220$ & $1.80 \times 10^{-3}$ & 0.54 & 14 \\
$220-275$ & $4.13 \times 10^{-3}$ & 0.37 & 2.7 \\
$275-340$ & $3.16 \times 10^{-3}$ & 0.34 & 3.5 \\
$340-410$ & $2.00 \times 10^{-3}$ & 0.49 & 4.1 \\
$410-485$ & $1.18 \times 10^{-3}$ & 0.69 & 4.2 \\
$485-570$ & $6.73 \times 10^{-4}$ & 0.89 & 4.9 \\
$570-660$ & $3.75 \times 10^{-4}$ & 1.2 & 5.5 \\
$660-760$ & $2.05 \times 10^{-4}$ & 1.5 & 5.2 \\
$760-870$ & $1.15 \times 10^{-4}$ & 1.9 & 7.2 \\
$870-990$ & $6.23 \times 10^{-5}$ & 2.5 & 5.6 \\
$990-1115$ & $3.28 \times 10^{-5}$ & 3.6 & 13 \\
$1115-1250$ & $1.79 \times 10^{-5}$ & 4.5 & 12 \\
$1250-1925$ & $4.78 \times 10^{-6}$ & 3.3 & 12 \\
\hline
\end{tabular}

Table 5. Results of the normalised differential cross sections with relative uncertainties in the combined channel with respect to $H_{\mathrm{T}}$.

\begin{tabular}{|cccc|}
\hline $\begin{array}{c}S_{\mathrm{T}} \\
(\mathrm{GeV})\end{array}$ & $\begin{array}{c}\frac{1}{\mathrm{~d} \sigma} \frac{\mathrm{d} \sigma}{\mathrm{d} S_{\mathrm{T}}} \\
\left(\mathrm{GeV}^{-1}\right)\end{array}$ & $\begin{array}{c}\text { Stat. unc. } \\
(\%)\end{array}$ & $\begin{array}{c}\text { Syst. unc. } \\
(\%)\end{array}$ \\
\hline $136-315$ & $9.99 \times 10^{-4}$ & 0.66 & 17 \\
$315-390$ & $3.49 \times 10^{-3}$ & 0.37 & 2.8 \\
$390-475$ & $2.57 \times 10^{-3}$ & 0.32 & 3.7 \\
$475-565$ & $1.54 \times 10^{-3}$ & 0.49 & 4.6 \\
$565-665$ & $8.52 \times 10^{-4}$ & 0.71 & 5.1 \\
$665-770$ & $4.68 \times 10^{-4}$ & 1.0 & 5.5 \\
$770-885$ & $2.54 \times 10^{-4}$ & 1.3 & 5.4 \\
$885-1010$ & $1.34 \times 10^{-4}$ & 1.8 & 6.4 \\
$1010-1140$ & $7.36 \times 10^{-5}$ & 2.5 & 6.1 \\
$1140-1285$ & $3.98 \times 10^{-5}$ & 3.2 & 11 \\
$1285-1440$ & $1.96 \times 10^{-5}$ & 4.6 & 9.9 \\
$1440-1615$ & $1.16 \times 10^{-5}$ & 5.3 & 17 \\
$1615-2490$ & $2.35 \times 10^{-6}$ & 4.6 & 14 \\
\hline
\end{tabular}

Table 6. Results of the normalised differential cross sections with relative uncertainties in the combined channel with respect to $S_{\mathrm{T}}$. 


\begin{tabular}{|cccc|}
\hline $\begin{array}{c}p_{\mathrm{T}}^{\text {miss }} \\
(\mathrm{GeV})\end{array}$ & $\begin{array}{c}\frac{1}{\mathrm{~d} \sigma} \frac{\mathrm{d} \sigma}{\mathrm{d} p_{\mathrm{T}}^{\mathrm{miss}}} \\
\left(\mathrm{GeV}^{-1}\right)\end{array}$ & $\begin{array}{c}\text { Stat. unc. } \\
(\%)\end{array}$ & $\begin{array}{c}\text { Syst. unc. } \\
(\%)\end{array}$ \\
\hline $0-50$ & $1.05 \times 10^{-2}$ & 0.16 & 3.7 \\
$50-105$ & $6.62 \times 10^{-3}$ & 0.24 & 2.9 \\
$105-175$ & $1.26 \times 10^{-3}$ & 0.75 & 9.1 \\
$175-245$ & $2.43 \times 10^{-4}$ & 2.0 & 4.4 \\
$245-315$ & $5.93 \times 10^{-5}$ & 4.5 & 11 \\
$315-565$ & $7.63 \times 10^{-6}$ & 5.8 & 12 \\
\hline
\end{tabular}

Table 7. Results of the normalised differential cross sections with relative uncertainties in the combined channel with respect to $p_{\mathrm{T}}^{\text {miss }}$.

\begin{tabular}{|cccc|}
\hline $\begin{array}{c}p_{\mathrm{T}}^{\mathrm{W}} \\
(\mathrm{GeV})\end{array}$ & $\begin{array}{c}\frac{1}{\mathrm{~d} \sigma} \frac{\mathrm{d} \sigma}{\mathrm{d} p_{\mathrm{T}}^{\mathrm{W}}} \\
\left(\mathrm{GeV}^{-1}\right)\end{array}$ & $\begin{array}{c}\text { Stat. unc. } \\
(\%)\end{array}$ & $\begin{array}{c}\text { Syst. unc. } \\
(\%)\end{array}$ \\
\hline $0-50$ & $5.12 \times 10^{-3}$ & 0.36 & 3.9 \\
$50-105$ & $7.44 \times 10^{-3}$ & 0.22 & 0.81 \\
$105-165$ & $3.72 \times 10^{-3}$ & 0.37 & 3.2 \\
$165-240$ & $1.09 \times 10^{-3}$ & 0.69 & 4.8 \\
$240-325$ & $2.59 \times 10^{-4}$ & 1.4 & 5.4 \\
$325-415$ & $6.32 \times 10^{-5}$ & 2.8 & 7.7 \\
$415-845$ & $5.47 \times 10^{-6}$ & 4.1 & 13 \\
\hline
\end{tabular}

Table 8. Results of the normalised differential cross sections with relative uncertainties in the combined channel with respect to $p_{\mathrm{T}}^{\mathrm{W}}$. 


\begin{tabular}{|cccc|}
\hline $\begin{array}{c}p_{\mathrm{T}}^{\ell} \\
(\mathrm{GeV})\end{array}$ & $\begin{array}{c}\frac{1}{\mathrm{~d} \sigma} \mathrm{d} \sigma \\
\mathrm{d} p_{\mathrm{T}}^{\ell}\end{array}$ & $\begin{array}{c}\text { Stat. unc. } \\
\left(\mathrm{GeV}^{-1}\right)\end{array}$ & $\begin{array}{c}\text { Syst. unc. } \\
(\%)\end{array}$ \\
\hline $26-40$ & $2.14 \times 10^{-2}$ & 0.30 & 1.2 \\
$40-55$ & $1.63 \times 10^{-2}$ & 0.26 & 0.89 \\
$55-70$ & $1.10 \times 10^{-2}$ & 0.32 & 0.69 \\
$70-85$ & $7.08 \times 10^{-3}$ & 0.41 & 1.0 \\
$85-100$ & $4.40 \times 10^{-3}$ & 0.52 & 1.4 \\
$100-115$ & $2.74 \times 10^{-3}$ & 0.68 & 1.8 \\
$115-130$ & $1.79 \times 10^{-3}$ & 0.86 & 3.0 \\
$130-145$ & $1.13 \times 10^{-3}$ & 1.1 & 2.6 \\
$145-160$ & $7.21 \times 10^{-4}$ & 1.4 & 2.6 \\
$160-175$ & $4.76 \times 10^{-4}$ & 1.9 & 4.2 \\
$175-190$ & $3.31 \times 10^{-4}$ & 2.3 & 7.4 \\
$190-205$ & $2.17 \times 10^{-4}$ & 3.0 & 6.3 \\
$205-220$ & $1.51 \times 10^{-4}$ & 3.8 & 8.8 \\
$220-235$ & $1.06 \times 10^{-4}$ & 4.7 & 9.6 \\
$235-255$ & $7.28 \times 10^{-5}$ & 4.6 & 13 \\
$255-285$ & $4.25 \times 10^{-5}$ & 4.5 & 11 \\
$285-435$ & $9.45 \times 10^{-6}$ & 4.4 & 13 \\
\hline
\end{tabular}

Table 9. Results of the normalised differential cross sections with relative uncertainties in the combined channel with respect to $p_{\mathrm{T}}^{\ell}$.

\begin{tabular}{|cccc|}
\hline$\left|\eta^{\ell}\right|$ & $\frac{1}{\mathrm{~d} \sigma} \frac{\mathrm{d} \sigma}{\mathrm{d}\left|\eta^{\ell}\right|}$ & $\begin{array}{c}\text { Stat. unc. } \\
(\%)\end{array}$ & $\begin{array}{c}\text { Syst. unc. } \\
(\%)\end{array}$ \\
\hline $0.00-0.30$ & 0.648 & 0.28 & 1.0 \\
$0.30-0.60$ & 0.622 & 0.28 & 0.89 \\
$0.60-0.90$ & 0.563 & 0.30 & 0.86 \\
$0.90-1.20$ & 0.490 & 0.33 & 0.69 \\
$1.20-1.50$ & 0.388 & 0.40 & 1.1 \\
$1.50-1.80$ & 0.288 & 0.54 & 1.9 \\
$1.80-2.00$ & 0.213 & 0.74 & 3.0 \\
$2.00-2.40$ & 0.144 & 1.0 & 4.4 \\
\hline
\end{tabular}

Table 10. Results of the normalised differential cross sections with relative uncertainties in the combined channel with respect to $\left|\eta^{\ell}\right|$. 


\section{B Tabulated absolute differential t $\mathrm{t} \overline{\mathrm{t}}$ production cross sections}

\begin{tabular}{|cccc|}
\hline$N_{\text {jets }}$ & $\begin{array}{c}\mathrm{d} \sigma \\
\mathrm{d} N_{\text {jets }} \\
(\mathrm{pb})\end{array}$ & $\begin{array}{c}\text { Stat. unc. } \\
(\%)\end{array}$ & $\begin{array}{c}\text { Syst. unc. } \\
(\%)\end{array}$ \\
\hline $3.5-4.5$ & 40.6 & 0.33 & 9.3 \\
$4.5-5.5$ & 31.9 & 0.29 & 8.7 \\
$5.5-6.5$ & 16.8 & 0.47 & 9.9 \\
$6.5-7.5$ & 7.22 & 1.0 & 11 \\
$7.5-8.5$ & 2.60 & 3.0 & 13 \\
$8.5-10.5$ & 0.591 & 3.9 & 12 \\
\hline
\end{tabular}

Table 11. Results of the absolute differential cross sections with relative uncertainties in the combined channel with respect to $N_{\text {jets }}$.

\begin{tabular}{|cccc|}
\hline $\begin{array}{c}H_{\mathrm{T}} \\
(\mathrm{GeV})\end{array}$ & $\begin{array}{c}\frac{\mathrm{d} \sigma}{\mathrm{d} H_{\mathrm{T}}} \\
\left(\mathrm{pb} \mathrm{GeV}^{-1}\right)\end{array}$ & $\begin{array}{c}\text { Stat. unc. } \\
(\%)\end{array}$ & $\begin{array}{c}\text { Syst. unc. } \\
(\%)\end{array}$ \\
\hline $110-220$ & 0.184 & 0.62 & 15 \\
$220-275$ & 0.424 & 0.38 & 9.7 \\
$275-340$ & 0.324 & 0.35 & 10 \\
$340-410$ & 0.205 & 0.50 & 10 \\
$410-485$ & 0.121 & 0.70 & 10 \\
$485-570$ & $6.91 \times 10^{-2}$ & 0.89 & 11 \\
$570-660$ & $3.85 \times 10^{-2}$ & 1.2 & 11 \\
$660-760$ & $2.10 \times 10^{-2}$ & 1.5 & 10 \\
$760-870$ & $1.18 \times 10^{-2}$ & 2.0 & 12 \\
$870-990$ & $6.39 \times 10^{-3}$ & 2.5 & 11 \\
$990-1115$ & $3.37 \times 10^{-3}$ & 3.6 & 16 \\
$1115-1250$ & $1.84 \times 10^{-3}$ & 4.5 & 14 \\
$1250-1925$ & $4.91 \times 10^{-4}$ & 3.3 & 16 \\
\hline
\end{tabular}

Table 12. Results of the absolute differential cross sections with relative uncertainties in the combined channel with respect to $H_{\mathrm{T}}$. 


\begin{tabular}{|cccc|}
\hline $\begin{array}{c}S_{\mathrm{T}} \\
(\mathrm{GeV})\end{array}$ & $\begin{array}{c}\frac{\mathrm{d} \sigma}{\mathrm{d} S_{\mathrm{T}}} \\
\left(\mathrm{pb} \mathrm{GeV}^{-1}\right)\end{array}$ & $\begin{array}{c}\text { Stat. unc. } \\
(\%)\end{array}$ & $\begin{array}{c}\text { Syst. unc. } \\
(\%)\end{array}$ \\
\hline $136-315$ & 0.103 & 0.73 & 18 \\
$315-390$ & 0.358 & 0.38 & 9.5 \\
$390-475$ & 0.264 & 0.34 & 10 \\
$475-565$ & 0.158 & 0.50 & 10 \\
$565-665$ & $8.76 \times 10^{-2}$ & 0.71 & 11 \\
$665-770$ & $4.81 \times 10^{-2}$ & 1.0 & 11 \\
$770-885$ & $2.61 \times 10^{-2}$ & 1.3 & 11 \\
$885-1010$ & $1.38 \times 10^{-2}$ & 1.8 & 11 \\
$1010-1140$ & $7.56 \times 10^{-3}$ & 2.5 & 11 \\
$1140-1285$ & $4.09 \times 10^{-3}$ & 3.2 & 15 \\
$1285-1440$ & $2.02 \times 10^{-3}$ & 4.6 & 13 \\
$1440-1615$ & $1.20 \times 10^{-3}$ & 5.3 & 20 \\
$1615-2490$ & $2.42 \times 10^{-4}$ & 4.6 & 18 \\
\hline
\end{tabular}

Table 13. Results of the absolute differential cross sections with relative uncertainties in the combined channel with respect to $S_{\mathrm{T}}$.

\begin{tabular}{|cccc|}
\hline $\begin{array}{c}p_{\mathrm{T}}^{\text {miss }} \\
(\mathrm{GeV})\end{array}$ & $\begin{array}{c}\frac{\mathrm{d} \sigma}{\mathrm{d} p_{\mathrm{T}}^{\text {miss }}} \\
\left(\mathrm{pb} \mathrm{GeV}^{-1}\right)\end{array}$ & $\begin{array}{c}\text { Stat. unc. } \\
(\%)\end{array}$ & $\begin{array}{c}\text { Syst. unc. } \\
(\%)\end{array}$ \\
\hline $0-50$ & 1.05 & 0.21 & 8.8 \\
$50-105$ & 0.664 & 0.28 & 10 \\
$105-175$ & 0.126 & 0.76 & 15 \\
$175-245$ & $2.44 \times 10^{-2}$ & 2.0 & 11 \\
$245-315$ & $5.96 \times 10^{-3}$ & 4.5 & 15 \\
$315-565$ & $7.66 \times 10^{-4}$ & 5.8 & 17 \\
\hline
\end{tabular}

Table 14. Results of the absolute differential cross sections with relative uncertainties in the combined channel with respect to $p_{\mathrm{T}}^{\text {miss }}$. 


\begin{tabular}{|cccc|}
\hline $\begin{array}{c}p_{\mathrm{T}}^{\mathrm{W}} \\
(\mathrm{GeV})\end{array}$ & $\begin{array}{c}\frac{\mathrm{d} \sigma}{\mathrm{d} p_{\mathrm{T}}^{\mathrm{W}}} \\
\left(\mathrm{pb} \mathrm{GeV}^{-1}\right)\end{array}$ & $\begin{array}{c}\text { Stat. unc. } \\
(\%)\end{array}$ & $\begin{array}{c}\text { Syst. unc. } \\
(\%)\end{array}$ \\
\hline $0-50$ & 0.518 & 0.41 & 8.3 \\
$50-105$ & 0.752 & 0.25 & 8.7 \\
$105-165$ & 0.377 & 0.39 & 11 \\
$165-240$ & 0.111 & 0.70 & 11 \\
$240-325$ & $2.62 \times 10^{-2}$ & 1.4 & 12 \\
$325-415$ & $6.40 \times 10^{-3}$ & 2.8 & 13 \\
$415-845$ & $5.54 \times 10^{-4}$ & 4.1 & 17 \\
\hline
\end{tabular}

Table 15. Results of the absolute differential cross sections with relative uncertainties in the combined channel with respect to $p_{\mathrm{T}}^{\mathrm{W}}$.

\begin{tabular}{|cccc|}
\hline $\begin{array}{c}p_{\mathrm{T}}^{\ell} \\
(\mathrm{GeV})\end{array}$ & $\begin{array}{c}\frac{\mathrm{d} \sigma}{\mathrm{d} p_{\mathrm{T}}^{\ell}} \\
\left(\mathrm{pb} \mathrm{GeV}^{-1}\right)\end{array}$ & $\begin{array}{c}\text { Stat. unc. } \\
(\%)\end{array}$ & $\begin{array}{c}\text { Syst. unc. } \\
(\%)\end{array}$ \\
\hline $26-40$ & 2.16 & 0.39 & 9.5 \\
$40-55$ & 1.64 & 0.28 & 9.2 \\
$55-70$ & 1.11 & 0.33 & 9.1 \\
$70-85$ & 0.715 & 0.41 & 8.8 \\
$85-100$ & 0.443 & 0.53 & 8.9 \\
$100-115$ & 0.277 & 0.68 & 8.7 \\
$115-130$ & 0.181 & 0.86 & 9.0 \\
$130-145$ & 0.114 & 1.1 & 8.6 \\
$145-160$ & $7.28 \times 10^{-2}$ & 1.4 & 8.8 \\
$160-175$ & $4.80 \times 10^{-2}$ & 1.9 & 9.0 \\
$175-190$ & $3.34 \times 10^{-2}$ & 2.3 & 11 \\
$190-205$ & $2.19 \times 10^{-2}$ & 3.0 & 9.8 \\
$205-220$ & $1.52 \times 10^{-2}$ & 3.8 & 12 \\
$220-235$ & $1.07 \times 10^{-2}$ & 4.7 & 13 \\
$235-255$ & $7.34 \times 10^{-3}$ & 4.6 & 16 \\
$255-285$ & $4.29 \times 10^{-3}$ & 4.5 & 14 \\
$285-435$ & $9.53 \times 10^{-4}$ & 4.4 & 16 \\
\hline
\end{tabular}

Table 16. Results of the absolute differential cross sections with relative uncertainties in the combined channel with respect to $p_{\mathrm{T}}^{\ell}$. 


\begin{tabular}{|cccc|}
\hline$\left|\eta^{\ell}\right|$ & $\begin{array}{c}\mathrm{d} \sigma \\
\mathrm{d}\left|\eta^{\ell}\right| \\
(\mathrm{pb})\end{array}$ & $\begin{array}{c}\text { Stat. unc. } \\
(\%)\end{array}$ & $\begin{array}{c}\text { Syst. unc. } \\
(\%)\end{array}$ \\
\hline $0.00-0.30$ & 65.5 & 0.31 & 8.9 \\
$0.30-0.60$ & 62.9 & 0.30 & 8.8 \\
$0.60-0.90$ & 56.9 & 0.32 & 8.8 \\
$0.90-1.20$ & 49.5 & 0.35 & 9.0 \\
$1.20-1.50$ & 39.2 & 0.43 & 9.1 \\
$1.50-1.80$ & 29.1 & 0.57 & 9.5 \\
$1.80-2.00$ & 21.6 & 0.76 & 10 \\
$2.00-2.40$ & 14.6 & 1.1 & 11 \\
\hline
\end{tabular}

Table 17. Results of the absolute differential cross sections with relative uncertainties in the combined channel with respect to $\left|\eta^{\ell}\right|$.

\section{Tabulated minimum and maximum relative uncertainties for absolute cross sections}


Open Access. This article is distributed under the terms of the Creative Commons Attribution License (CC-BY 4.0), which permits any use, distribution and reproduction in any medium, provided the original author(s) and source are credited.

\section{References}

[1] CMS collaboration, The CMS experiment at the CERN LHC, 2008 JINST 3 S08004 [INSPIRE].

[2] CMS collaboration, Measurement of differential top-quark pair production cross sections in pp collisions at $\sqrt{s}=7 \mathrm{TeV}$, Eur. Phys. J. C 73 (2013) 2339 [arXiv:1211.2220] [INSPIRE].

[3] CMS collaboration, Measurement of the differential cross section for top quark pair production in pp collisions at $\sqrt{s}=8 \mathrm{TeV}$, Eur. Phys. J. C 75 (2015) 542 [arXiv: 1505.04480] [INSPIRE].

[4] CMS collaboration, Measurement of double-differential cross sections for top quark pair production in pp collisions at $\sqrt{s}=8 \mathrm{TeV}$ and impact on parton distribution functions, Eur. Phys. J. C 77 (2017) 459 [arXiv:1703.01630] [InSPIRE].

[5] CMS collaboration, Measurement of the integrated and differential t $\bar{t}$ production cross sections for high-p top quarks in pp collisions at $\sqrt{s}=8 \mathrm{TeV}$, Phys. Rev. D 94 (2016) 072002 [arXiv: 1605.00116] [INSPIRE].

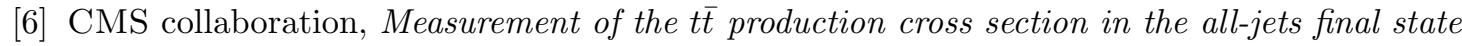
in pp collisions at $\sqrt{s}=8 \mathrm{TeV}$, Eur. Phys. J. C 76 (2016) 128 [arXiv:1509.06076] [INSPIRE].

[7] CMS collaboration, Measurement of differential cross sections for top quark pair production using the lepton+jets final state in proton-proton collisions at $13 \mathrm{TeV}$, Phys. Rev. D 95 (2017) 092001 [arXiv: 1610.04191] [INSPIRE].

[8] CMS collaboration, Measurement of normalized differential t $\bar{t}$ cross sections in the dilepton channel from pp collisions at $\sqrt{s}=13 \mathrm{TeV}$, JHEP 04 (2018) 060 [arXiv:1708.07638] [INSPIRE].

[9] ATLAS collaboration, Measurements of top-quark pair differential cross-sections in the lepton+jets channel in pp collisions at $\sqrt{s}=13$ TeV using the ATLAS detector, JHEP 11 (2017) 191 [arXiv: 1708.00727] [INSPIRE].

[10] ATLAS collaboration, Measurements of top-quark pair differential cross-sections in the e $\mu$ channel in pp collisions at $\sqrt{s}=13 \mathrm{TeV}$ using the ATLAS detector, Eur. Phys. J. C 77 (2017) 292 [arXiv : 1612.05220] [INSPIRE].

[11] CMS collaboration, Measurement of the differential cross sections for top quark pair production as a function of kinematic event variables in pp collisions at $\sqrt{s}=7$ and $8 \mathrm{TeV}$, Phys. Rev. D 94 (2016) 052006 [arXiv:1607.00837] [InSPIRE].

[12] CMS collaboration, The CMS trigger system, 2017 JINST 12 P01020 [arXiv:1609.02366] [INSPIRE].

[13] S. Frixione, P. Nason and C. Oleari, Matching NLO QCD computations with parton shower simulations: the POWHEG method, JHEP 11 (2007) 070 [arXiv:0709.2092] [INSPIRE].

[14] P. Nason, A new method for combining NLO QCD with shower Monte Carlo algorithms, JHEP 11 (2004) 040 [hep-ph/0409146] [INSPIRE]. 
[15] S. Alioli, P. Nason, C. Oleari and E. Re, A general framework for implementing NLO calculations in shower Monte Carlo programs: the POWHEG BOX, JHEP 06 (2010) 043 [arXiv: 1002.2581] [INSPIRE].

[16] S. Frixione, P. Nason and G. Ridolfi, A positive-weight next-to-leading-order Monte Carlo for heavy flavour hadroproduction, JHEP 09 (2007) 126 [arXiv:0707.3088] [INSPIRE].

[17] T. Sjöstrand, S. Mrenna and P.Z. Skands, PYTHIA 6.4 physics and manual, JHEP 05 (2006) 026 [hep-ph/0603175] [INSPIRE].

[18] T. Sjöstrand, S. Mrenna and P.Z. Skands, A brief introduction to PYTHIA 8.1, Comput. Phys. Commun. 178 (2008) 852 [arXiv:0710.3820] [INSPIRE].

[19] CMS collaboration, Investigations of the impact of the parton shower tuning in PYTHIA 8 in the modelling of $t \bar{t}$ at $\sqrt{s}=8$ and $13 \mathrm{TeV}$, CMS-PAS-TOP-16-021, CERN, Geneva Switzerland, (2016).

[20] M. Bahr et al., HERWIG++ physics and manual, Eur. Phys. J. C 58 (2008) 639 [arXiv:0803.0883] [INSPIRE].

[21] S. Gieseke, C. Rohr and A. Siodmok, Colour reconnections in HERWIG++, Eur. Phys. J. C 72 (2012) 2225 [arXiv:1206.0041] [INSPIRE].

[22] J. Alwall et al., The automated computation of tree-level and next-to-leading order differential cross sections and their matching to parton shower simulations, JHEP 07 (2014) 079 [arXiv: 1405.0301] [INSPIRE].

[23] CMS collaboration, Event generator tunes obtained from underlying event and multiparton scattering measurements, Eur. Phys. J. C 76 (2016) 155 [arXiv:1512.00815] [INSPIRE].

[24] J. Alwall et al., Comparative study of various algorithms for the merging of parton showers and matrix elements in hadronic collisions, Eur. Phys. J. C 53 (2008) 473 [arXiv:0706.2569] [INSPIRE].

[25] R. Frederix and S. Frixione, Merging meets matching in MC@NLO, JHEP 12 (2012) 061 [arXiv:1209.6215] [INSPIRE].

[26] NNPDF collaboration, R.D. Ball et al., Parton distributions for the LHC run II, JHEP 04 (2015) 040 [arXiv: 1410.8849] [INSPIRE].

[27] M. Beneke, P. Falgari, S. Klein and C. Schwinn, Hadronic top-quark pair production with NNLL threshold resummation, Nucl. Phys. B 855 (2012) 695 [arXiv:1109.1536] [INSPIRE].

[28] M. Cacciari, M. Czakon, M. Mangano, A. Mitov and P. Nason, Top-pair production at hadron colliders with next-to-next-to-leading logarithmic soft-gluon resummation, Phys. Lett. B 710 (2012) 612 [arXiv:1111.5869] [INSPIRE].

[29] P. Bärnreuther, M. Czakon and A. Mitov, Percent level precision physics at the Tevatron: first genuine NNLO QCD corrections to $q \bar{q} \rightarrow t \bar{t}+X$, Phys. Rev. Lett. 109 (2012) 132001 [arXiv:1204.5201] [INSPIRE].

[30] M. Czakon and A. Mitov, NNLO corrections to top-pair production at hadron colliders: the all-fermionic scattering channels, JHEP 12 (2012) 054 [arXiv:1207.0236] [INSPIRE].

[31] M. Czakon and A. Mitov, NNLO corrections to top pair production at hadron colliders: the quark-gluon reaction, JHEP 01 (2013) 080 [arXiv: 1210.6832] [INSPIRE].

[32] M. Czakon, P. Fiedler and A. Mitov, Total top-quark pair-production cross section at hadron colliders through $O\left(\alpha_{S}^{4}\right)$, Phys. Rev. Lett. 110 (2013) 252004 [arXiv:1303.6254] [INSPIRE]. 
[33] M. Czakon and A. Mitov, Top++: a program for the calculation of the top-pair cross-section at hadron colliders, Comput. Phys. Commun. 185 (2014) 2930 [arXiv:1112.5675] [InSPIRE].

[34] M. Aliev, H. Lacker, U. Langenfeld, S. Moch, P. Uwer and M. Wiedermann, HATHOR: HAdronic Top and Heavy quarks crOss section calculatoR, Comput. Phys. Commun. 182 (2011) 1034 [arXiv: 1007.1327] [INSPIRE].

[35] P. Kant et al., HATHOR for single top-quark production: updated predictions and uncertainty estimates for single top-quark production in hadronic collisions, Comput. Phys. Commun. 191 (2015) 74 [arXiv:1406.4403] [INSPIRE].

[36] R. Frederix, E. Re and P. Torrielli, Single-top t-channel hadroproduction in the four-flavour scheme with POWHEG and aMC@NLO, JHEP 09 (2012) 130 [arXiv:1207.5391] [INSPIRE].

[37] S. Alioli, P. Nason, C. Oleari and E. Re, NLO single-top production matched with shower in POWHEG: s- and t-channel contributions, JHEP 09 (2009) 111 [Erratum ibid. 02 (2010) 011] [arXiv: 0907.4076] [INSPIRE].

[38] E. Re, Single-top Wt-channel production matched with parton showers using the POWHEG method, Eur. Phys. J. C 71 (2011) 1547 [arXiv: 1009. 2450] [INSPIRE].

[39] S. Frixione, E. Laenen, P. Motylinski, B.R. Webber and C.D. White, Single-top hadroproduction in association with a $W$ boson, JHEP 07 (2008) 029 [arXiv:0805.3067] [INSPIRE].

[40] Y. Li and F. Petriello, Combining QCD and electroweak corrections to dilepton production in FEWZ, Phys. Rev. D 86 (2012) 094034 [arXiv: 1208.5967] [INSPIRE].

[41] GEANT4 collaboration, S. Agostinelli et al., GEANT4: a simulation toolkit, Nucl. Instrum. Meth. A 506 (2003) 250 [INSPIRE].

[42] CMS collaboration, Particle-flow reconstruction and global event description with the CMS detector, 2017 JINST 12 P10003 [arXiv:1706.04965] [INSPIRE].

[43] CMS collaboration, Performance of electron reconstruction and selection with the CMS detector in proton-proton collisions at $\sqrt{s}=8 \mathrm{TeV}, 2015$ JINST $10 \mathrm{P} 06005$ [arXiv: 1502.02701] [INSPIRE].

[44] CMS collaboration, Performance of CMS muon reconstruction in pp collision events at $\sqrt{s}=7 \mathrm{TeV}, 2012$ JINST 7 P10002 [arXiv:1206.4071] [INSPIRE].

[45] CMS collaboration, Measurement of the inclusive $W$ and $Z$ production cross sections in pp collisions at $\sqrt{s}=7 \mathrm{TeV}$ with the CMS experiment, JHEP 10 (2011) 132 [arXiv:1107.4789] [INSPIRE].

[46] M. Cacciari, G.P. Salam and G. Soyez, The anti- $k_{t}$ jet clustering algorithm, JHEP 04 (2008) 063 [arXiv: 0802.1189] [INSPIRE].

[47] M. Cacciari, G.P. Salam and G. Soyez, FastJet user manual, Eur. Phys. J. C 72 (2012) 1896 [arXiv: 1111.6097] [INSPIRE].

[48] CMS collaboration, Jet energy scale and resolution in the CMS experiment in pp collisions at $8 \mathrm{TeV}, 2017$ JINST $12 \mathrm{P} 02014$ [arXiv: 1607.03663] [INSPIRE].

[49] CMS collaboration, Identification of b-quark jets with the CMS experiment, 2013 JINST 8 P04013 [arXiv: 1211.4462] [INSPIRE].

[50] CMS collaboration, Identification of heavy-flavour jets with the CMS detector in $p p$ collisions at $13 \mathrm{TeV}, 2018$ JINST 13 P05011 [arXiv:1712.07158] [INSPIRE]. 
[51] A. Buckley et al., Rivet user manual, Comput. Phys. Commun. 184 (2013) 2803 [arXiv: 1003.0694] [INSPIRE].

[52] CMS collaboration, Object definitions for top quark analyses at the particle level, CMS-NOTE-2017-004, CERN, Geneva Switzerland, (2017).

[53] S. Schmitt, TUnfold: an algorithm for correcting migration effects in high energy physics, 2012 JINST 7 T10003 [arXiv:1205.6201] [INSPIRE].

[54] CMS collaboration, CMS luminosity measurements for the 2016 data taking period, CMS-PAS-LUM-17-001, CERN, Geneva Switzerland, (2017).

[55] CMS collaboration, Measurement of the inelastic proton-proton cross section at $\sqrt{s}=13 \mathrm{TeV}$, arXiv:1802.02613 [INSPIRE].

[56] CMS collaboration, Cross section measurement of $t$-channel single top quark production in pp collisions at $\sqrt{s}=13 \mathrm{TeV}$, Phys. Lett. B 772 (2017) 752 [arXiv:1610.00678] [InSPIRE].

[57] CMS collaboration, Measurement of the production cross section of a $W$ boson in association with two b jets in pp collisions at $\sqrt{s}=8 \mathrm{TeV}$, Eur. Phys. J. C 77 (2017) 92 [arXiv: 1608.07561] [INSPIRE].

[58] CMS collaboration, Measurements of the associated production of a $Z$ boson and $b$ jets in $p p$ collisions at $\sqrt{s}=8 \mathrm{TeV}$, Eur. Phys. J. C 77 (2017) 751 [arXiv:1611.06507] [INSPIRE].

[59] Particle Data Group collaboration, C. Patrignani et al., Review of particle physics, Chin. Phys. C 40 (2016) 100001 [INSPIRE].

[60] P. Skands, S. Carrazza and J. Rojo, Tuning PYTHIA 8.1: the Monash 2013 tune, Eur. Phys. J. C 74 (2014) 3024 [arXiv: 1404.5630] [INSPIRE].

[61] C. Peterson, D. Schlatter, I. Schmitt and P.M. Zerwas, Scaling violations in inclusive $e^{+} e^{-}$ annihilation spectra, Phys. Rev. D 27 (1983) 105 [INSPIRE].

[62] J.R. Christiansen and P.Z. Skands, String formation beyond leading colour, JHEP 08 (2015) 003 [arXiv: 1505.01681] [INSPIRE].

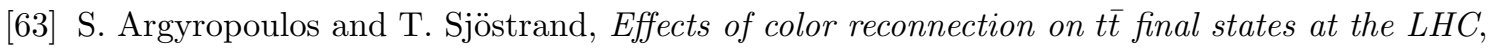
JHEP 11 (2014) 043 [arXiv: 1407.6653] [InSPIRE]. 


\section{The CMS collaboration}

\section{Yerevan Physics Institute, Yerevan, Armenia}

A.M. Sirunyan, A. Tumasyan

\section{Institut für Hochenergiephysik, Wien, Austria}

W. Adam, F. Ambrogi, E. Asilar, T. Bergauer, J. Brandstetter, E. Brondolin, M. Dragicevic, J. Erö, A. Escalante Del Valle, M. Flechl, M. Friedl, R. Frühwirth ${ }^{1}$, V.M. Ghete, J. Grossmann, J. Hrubec, M. Jeitler ${ }^{1}$, A. König, N. Krammer, I. Krätschmer, D. Liko, T. Madlener, I. Mikulec, E. Pree, N. Rad, H. Rohringer, J. Schieck ${ }^{1}$, R. Schöfbeck, M. Spanring, D. Spitzbart, A. Taurok, W. Waltenberger, J. Wittmann, C.-E. Wulz ${ }^{1}$, M. Zarucki

\section{Institute for Nuclear Problems, Minsk, Belarus}

V. Chekhovsky, V. Mossolov, J. Suarez Gonzalez

\section{Universiteit Antwerpen, Antwerpen, Belgium}

E.A. De Wolf, D. Di Croce, X. Janssen, J. Lauwers, M. Pieters, M. Van De Klundert, H. Van Haevermaet, P. Van Mechelen, N. Van Remortel

\section{Vrije Universiteit Brussel, Brussel, Belgium}

S. Abu Zeid, F. Blekman, J. D'Hondt, I. De Bruyn, J. De Clercq, K. Deroover, G. Flouris, D. Lontkovskyi, S. Lowette, I. Marchesini, S. Moortgat, L. Moreels, Q. Python, K. Skovpen, S. Tavernier, W. Van Doninck, P. Van Mulders, I. Van Parijs

\section{Université Libre de Bruxelles, Bruxelles, Belgium}

D. Beghin, B. Bilin, H. Brun, B. Clerbaux, G. De Lentdecker, H. Delannoy, B. Dorney, G. Fasanella, L. Favart, R. Goldouzian, A. Grebenyuk, A.K. Kalsi, T. Lenzi, J. Luetic, T. Maerschalk, T. Seva, E. Starling, C. Vander Velde, P. Vanlaer, D. Vannerom, R. Yonamine, F. Zenoni

\section{Ghent University, Ghent, Belgium}

T. Cornelis, D. Dobur, A. Fagot, M. Gul, I. Khvastunov², D. Poyraz, C. Roskas, D. Trocino, M. Tytgat, W. Verbeke, M. Vit, N. Zaganidis

\section{Université Catholique de Louvain, Louvain-la-Neuve, Belgium}

H. Bakhshiansohi, O. Bondu, S. Brochet, G. Bruno, C. Caputo, A. Caudron, P. David, S. De Visscher, C. Delaere, M. Delcourt, B. Francois, A. Giammanco, G. Krintiras, V. Lemaitre, A. Magitteri, A. Mertens, M. Musich, K. Piotrzkowski, L. Quertenmont, A. Saggio, M. Vidal Marono, S. Wertz, J. Zobec

\section{Centro Brasileiro de Pesquisas Fisicas, Rio de Janeiro, Brazil}

W.L. Aldá Júnior, F.L. Alves, G.A. Alves, L. Brito, G. Correia Silva, C. Hensel, A. Moraes, M.E. Pol, P. Rebello Teles

\section{Universidade do Estado do Rio de Janeiro, Rio de Janeiro, Brazil}

E. Belchior Batista Das Chagas, W. Carvalho, J. Chinellato ${ }^{3}$, E. Coelho, E.M. Da Costa, G.G. Da Silveira ${ }^{4}$, D. De Jesus Damiao, S. Fonseca De Souza, L.M. Huertas 
Guativa, H. Malbouisson, M. Melo De Almeida, C. Mora Herrera, L. Mundim, H. Nogima, L.J. Sanchez Rosas, A. Santoro, A. Sznajder, M. Thiel, E.J. Tonelli Manganote ${ }^{3}$, F. Torres Da Silva De Araujo, A. Vilela Pereira

Universidade Estadual Paulista $^{a}$, Universidade Federal do ABC ${ }^{b}$, São Paulo, Brazil

S. Ahuja $^{a}$, C.A. Bernardes ${ }^{a}$, T.R. Fernandez Perez Tomei ${ }^{a}$, E.M. Gregores ${ }^{b}$, P.G. Mercadante ${ }^{b}$, S.F. Novaes ${ }^{a}$, Sandra S. Padula ${ }^{a}$, D. Romero Abad ${ }^{b}$, J.C. Ruiz Vargas ${ }^{a}$

Institute for Nuclear Research and Nuclear Energy, Bulgarian Academy of Sciences, Sofia, Bulgaria

A. Aleksandrov, R. Hadjiiska, P. Iaydjiev, A. Marinov, M. Misheva, M. Rodozov, M. Shopova, G. Sultanov

University of Sofia, Sofia, Bulgaria

A. Dimitrov, L. Litov, B. Pavlov, P. Petkov

Beihang University, Beijing, China

W. Fang ${ }^{5}$, X. Gao ${ }^{5}$, L. Yuan

Institute of High Energy Physics, Beijing, China

M. Ahmad, J.G. Bian, G.M. Chen, H.S. Chen, M. Chen, Y. Chen, C.H. Jiang, D. Leggat, H. Liao, Z. Liu, F. Romeo, S.M. Shaheen, A. Spiezia, J. Tao, C. Wang, Z. Wang, E. Yazgan, H. Zhang, J. Zhao

State Key Laboratory of Nuclear Physics and Technology, Peking University, Beijing, China

Y. Ban, G. Chen, J. Li, Q. Li, S. Liu, Y. Mao, S.J. Qian, D. Wang, Z. Xu

Tsinghua University, Beijing, China

Y. Wang

Universidad de Los Andes, Bogota, Colombia

C. Avila, A. Cabrera, C.A. Carrillo Montoya, L.F. Chaparro Sierra, C. Florez, C.F. González Hernández, J.D. Ruiz Alvarez, M.A. Segura Delgado

University of Split, Faculty of Electrical Engineering, Mechanical Engineering and Naval Architecture, Split, Croatia

B. Courbon, N. Godinovic, D. Lelas, I. Puljak, P.M. Ribeiro Cipriano, T. Sculac

University of Split, Faculty of Science, Split, Croatia

Z. Antunovic, M. Kovac

Institute Rudjer Boskovic, Zagreb, Croatia

V. Brigljevic, D. Ferencek, K. Kadija, B. Mesic, A. Starodumov ${ }^{6}$, T. Susa

University of Cyprus, Nicosia, Cyprus

M.W. Ather, A. Attikis, G. Mavromanolakis, J. Mousa, C. Nicolaou, F. Ptochos, P.A. Razis, H. Rykaczewski 
Charles University, Prague, Czech Republic

M. Finger ${ }^{7}$, M. Finger Jr. ${ }^{7}$

Universidad San Francisco de Quito, Quito, Ecuador

E. Carrera Jarrin

Academy of Scientific Research and Technology of the Arab Republic of Egypt, Egyptian Network of High Energy Physics, Cairo, Egypt

H. Abdalla ${ }^{8}$, A.A. Abdelalim ${ }^{9,10}$, S. Khalil ${ }^{10}$

National Institute of Chemical Physics and Biophysics, Tallinn, Estonia

S. Bhowmik, R.K. Dewanjee, M. Kadastik, L. Perrini, M. Raidal, C. Veelken

Department of Physics, University of Helsinki, Helsinki, Finland

P. Eerola, H. Kirschenmann, J. Pekkanen, M. Voutilainen

Helsinki Institute of Physics, Helsinki, Finland

J. Havukainen, J.K. Heikkilä, T. Järvinen, V. Karimäki, R. Kinnunen, T. Lampén,

K. Lassila-Perini, S. Laurila, S. Lehti, T. Lindén, P. Luukka, T. Mäenpää, H. Siikonen,

E. Tuominen, J. Tuominiemi

Lappeenranta University of Technology, Lappeenranta, Finland

T. Tuuva

IRFU, CEA, Université Paris-Saclay, Gif-sur-Yvette, France

M. Besancon, F. Couderc, M. Dejardin, D. Denegri, J.L. Faure, F. Ferri, S. Ganjour, S. Ghosh, A. Givernaud, P. Gras, G. Hamel de Monchenault, P. Jarry, C. Leloup, E. Locci, M. Machet, J. Malcles, G. Negro, J. Rander, A. Rosowsky, M.Ö. Sahin, M. Titov

Laboratoire Leprince-Ringuet, Ecole polytechnique, CNRS/IN2P3, Université Paris-Saclay, Palaiseau, France

A. Abdulsalam ${ }^{11}$, C. Amendola, I. Antropov, S. Baffioni, F. Beaudette, P. Busson,

L. Cadamuro, C. Charlot, R. Granier de Cassagnac, M. Jo, I. Kucher, S. Lisniak, A. Lobanov, J. Martin Blanco, M. Nguyen, C. Ochando, G. Ortona, P. Paganini, P. Pigard, R. Salerno, J.B. Sauvan, Y. Sirois, A.G. Stahl Leiton, Y. Yilmaz, A. Zabi, A. Zghiche

Université de Strasbourg, CNRS, IPHC UMR 7178, F-67000 Strasbourg, France

J.-L. Agram ${ }^{12}$, J. Andrea, D. Bloch, J.-M. Brom, M. Buttignol, E.C. Chabert, C. Collard, E. Conte ${ }^{12}$, X. Coubez, F. Drouhin ${ }^{12}$, J.-C. Fontaine ${ }^{12}$, D. Gelé, U. Goerlach, M. Jansová, P. Juillot, A.-C. Le Bihan, N. Tonon, P. Van Hove

Centre de Calcul de l'Institut National de Physique Nucleaire et de Physique des Particules, CNRS/IN2P3, Villeurbanne, France

S. Gadrat 
Université de Lyon, Université Claude Bernard Lyon 1, CNRS-IN2P3, Institut de Physique Nucléaire de Lyon, Villeurbanne, France

S. Beauceron, C. Bernet, G. Boudoul, N. Chanon, R. Chierici, D. Contardo, P. Depasse, H. El Mamouni, J. Fay, L. Finco, S. Gascon, M. Gouzevitch, G. Grenier, B. Ille, F. Lagarde, I.B. Laktineh, H. Lattaud, M. Lethuillier, L. Mirabito, A.L. Pequegnot, S. Perries, A. Popov ${ }^{13}$, V. Sordini, M. Vander Donckt, S. Viret, S. Zhang

Georgian Technical University, Tbilisi, Georgia

A. Khvedelidze ${ }^{7}$

Tbilisi State University, Tbilisi, Georgia

D. Lomidze

RWTH Aachen University, I. Physikalisches Institut, Aachen, Germany

C. Autermann, L. Feld, M.K. Kiesel, K. Klein, M. Lipinski, M. Preuten, C. Schomakers, J. Schulz, M. Teroerde, B. Wittmer, V. Zhukov ${ }^{13}$

RWTH Aachen University, III. Physikalisches Institut A, Aachen, Germany

A. Albert, D. Duchardt, M. Endres, M. Erdmann, S. Erdweg, T. Esch, R. Fischer, A. Güth,

T. Hebbeker, C. Heidemann, K. Hoepfner, S. Knutzen, M. Merschmeyer, A. Meyer,

P. Millet, S. Mukherjee, T. Pook, M. Radziej, H. Reithler, M. Rieger, F. Scheuch,

D. Teyssier, S. Thüer

RWTH Aachen University, III. Physikalisches Institut B, Aachen, Germany

G. Flügge, B. Kargoll, T. Kress, A. Künsken, T. Müller, A. Nehrkorn, A. Nowack, C. Pistone, O. Pooth, A. Stahl ${ }^{14}$

\section{Deutsches Elektronen-Synchrotron, Hamburg, Germany}

M. Aldaya Martin, T. Arndt, C. Asawatangtrakuldee, K. Beernaert, O. Behnke, U. Behrens, A. Bermúdez Martínez, A.A. Bin Anuar, K. Borras ${ }^{15}$, V. Botta, A. Campbell, P. Connor, C. Contreras-Campana, F. Costanza, A. De Wit, C. Diez Pardos, G. Eckerlin, D. Eckstein, T. Eichhorn, E. Eren, E. Gallo ${ }^{16}$, J. Garay Garcia, A. Geiser, J.M. Grados Luyando, A. Grohsjean, P. Gunnellini, M. Guthoff, A. Harb, J. Hauk, M. Hempel ${ }^{17}$, H. Jung, M. Kasemann, J. Keaveney, C. Kleinwort, I. Korol, D. Krücker, W. Lange, A. Lelek, T. Lenz, K. Lipka, W. Lohmann ${ }^{17}$, R. Mankel, I.-A. Melzer-Pellmann, A.B. Meyer, M. Meyer, M. Missiroli, G. Mittag, J. Mnich, A. Mussgiller, D. Pitzl, A. Raspereza, M. Savitskyi, P. Saxena, R. Shevchenko, N. Stefaniuk, H. Tholen, G.P. Van Onsem, R. Walsh, Y. Wen, K. Wichmann, C. Wissing, O. Zenaiev

University of Hamburg, Hamburg, Germany

R. Aggleton, S. Bein, V. Blobel, M. Centis Vignali, T. Dreyer, E. Garutti, D. Gonzalez, J. Haller, A. Hinzmann, M. Hoffmann, A. Karavdina, G. Kasieczka, R. Klanner, R. Kogler, N. Kovalchuk, S. Kurz, D. Marconi, J. Multhaup, M. Niedziela, D. Nowatschin, T. Peiffer, A. Perieanu, A. Reimers, C. Scharf, P. Schleper, A. Schmidt, S. Schumann, J. Schwandt, J. Sonneveld, H. Stadie, G. Steinbrück, F.M. Stober, M. Stöver, D. Troendle, E. Usai, A. Vanhoefer, B. Vormwald 
Institut für Experimentelle Teilchenphysik, Karlsruhe, Germany

M. Akbiyik, C. Barth, M. Baselga, S. Baur, E. Butz, R. Caspart, T. Chwalek, F. Colombo, W. De Boer, A. Dierlamm, N. Faltermann, B. Freund, R. Friese, M. Giffels, M.A. Harrendorf, F. Hartmann ${ }^{14}$, S.M. Heindl, U. Husemann, F. Kassel ${ }^{14}$, S. Kudella, H. Mildner, M.U. Mozer, Th. Müller, M. Plagge, G. Quast, K. Rabbertz, M. Schröder, I. Shvetsov, G. Sieber, H.J. Simonis, R. Ulrich, S. Wayand, M. Weber, T. Weiler, S. Williamson, C. Wöhrmann, R. Wolf

Institute of Nuclear and Particle Physics (INPP), NCSR Demokritos, Aghia Paraskevi, Greece

G. Anagnostou, G. Daskalakis, T. Geralis, A. Kyriakis, D. Loukas, I. Topsis-Giotis

National and Kapodistrian University of Athens, Athens, Greece

G. Karathanasis, S. Kesisoglou, A. Panagiotou, N. Saoulidou, E. Tziaferi

National Technical University of Athens, Athens, Greece

K. Kousouris, I. Papakrivopoulos

University of Ioánnina, Ioánnina, Greece

I. Evangelou, C. Foudas, P. Gianneios, P. Katsoulis, P. Kokkas, S. Mallios, N. Manthos, I. Papadopoulos, E. Paradas, J. Strologas, F.A. Triantis, D. Tsitsonis

MTA-ELTE Lendület CMS Particle and Nuclear Physics Group, Eötvös Loránd University, Budapest, Hungary

M. Csanad, N. Filipovic, G. Pasztor, O. Surányi, G.I. Veres ${ }^{18}$

Wigner Research Centre for Physics, Budapest, Hungary

G. Bencze, C. Hajdu, D. Horvath ${ }^{19}$, Á. Hunyadi, F. Sikler, V. Veszpremi, G. Vesztergombi ${ }^{18}$, T.Á. Vámi

Institute of Nuclear Research ATOMKI, Debrecen, Hungary

N. Beni, S. Czellar, J. Karancsi ${ }^{20}$, A. Makovec, J. Molnar, Z. Szillasi

Institute of Physics, University of Debrecen, Debrecen, Hungary

M. Bartók ${ }^{18}$, P. Raics, Z.L. Trocsanyi, B. Ujvari

Indian Institute of Science (IISc), Bangalore, India

S. Choudhury, J.R. Komaragiri

National Institute of Science Education and Research, Bhubaneswar, India

S. Bahinipati ${ }^{21}$, P. Mal, K. Mandal, A. Nayak ${ }^{22}$, D.K. Sahoo ${ }^{21}$, N. Sahoo, S.K. Swain

Panjab University, Chandigarh, India

S. Bansal, S.B. Beri, V. Bhatnagar, R. Chawla, N. Dhingra, R. Gupta, A. Kaur, M. Kaur, S. Kaur, R. Kumar, P. Kumari, A. Mehta, S. Sharma, J.B. Singh, G. Walia

University of Delhi, Delhi, India

Ashok Kumar, Aashaq Shah, A. Bhardwaj, S. Chauhan, B.C. Choudhary, R.B. Garg, S. Keshri, A. Kumar, S. Malhotra, M. Naimuddin, K. Ranjan, R. Sharma 
Saha Institute of Nuclear Physics, HBNI, Kolkata, India

R. Bhardwaj ${ }^{23}$, R. Bhattacharya, S. Bhattacharya, U. Bhawandeep ${ }^{23}$, D. Bhowmik, S. Dey, S. Dutt ${ }^{23}$, S. Dutta, S. Ghosh, N. Majumdar, A. Modak, K. Mondal, S. Mukhopadhyay, S. Nandan, A. Purohit, P.K. Rout, A. Roy, S. Roy Chowdhury, S. Sarkar, M. Sharan, B. Singh, S. Thakur ${ }^{23}$

\section{Indian Institute of Technology Madras, Madras, India}

P.K. Behera

Bhabha Atomic Research Centre, Mumbai, India

R. Chudasama, D. Dutta, V. Jha, V. Kumar, A.K. Mohanty ${ }^{14}$, P.K. Netrakanti, L.M. Pant, P. Shukla, A. Topkar

Tata Institute of Fundamental Research-A, Mumbai, India

T. Aziz, S. Dugad, B. Mahakud, S. Mitra, G.B. Mohanty, N. Sur, B. Sutar

Tata Institute of Fundamental Research-B, Mumbai, India

S. Banerjee, S. Bhattacharya, S. Chatterjee, P. Das, M. Guchait, Sa. Jain, S. Kumar, M. Maity ${ }^{24}$, G. Majumder, K. Mazumdar, T. Sarkar ${ }^{24}$, N. Wickramage ${ }^{25}$

Indian Institute of Science Education and Research (IISER), Pune, India

S. Chauhan, S. Dube, V. Hegde, A. Kapoor, K. Kothekar, S. Pandey, A. Rane, S. Sharma

Institute for Research in Fundamental Sciences (IPM), Tehran, Iran

S. Chenarani ${ }^{26}$, E. Eskandari Tadavani, S.M. Etesami ${ }^{26}$, M. Khakzad, M. Mohammadi Najafabadi, M. Naseri, S. Paktinat Mehdiabadi ${ }^{27}$, F. Rezaei Hosseinabadi, B. Safarzadeh ${ }^{28}$, M. Zeinali

University College Dublin, Dublin, Ireland

M. Felcini, M. Grunewald

INFN Sezione di Bari ${ }^{a}$, Università di Bari ${ }^{b}$, Politecnico di Bari ${ }^{c}$, Bari, Italy M. Abbrescia ${ }^{a, b}$, C. Calabria ${ }^{a, b}$, A. Colaleo ${ }^{a}$, D. Creanza ${ }^{a, c}$, L. Cristella ${ }^{a, b}$, N. De Filippis $^{a, c}$, M. De Palma ${ }^{a, b}$, A. Di Florio ${ }^{a, b}$, F. Errico ${ }^{a, b}$, L. Fiore $^{a}$, G. Iaselli ${ }^{a, c}$,

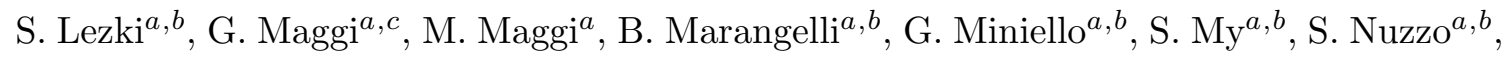
A. Pompili ${ }^{a, b}$, G. Pugliese ${ }^{a, c}$, R. Radogna ${ }^{a}$, A. Ranieri ${ }^{a}$, G. Selvaggi ${ }^{a, b}$, A. Sharma ${ }^{a}$, L. Silvestris ${ }^{a}, 14$, R. Venditti ${ }^{a}$, P. Verwilligen ${ }^{a}$, G. Zito ${ }^{a}$

INFN Sezione di Bologna ${ }^{a}$, Università di Bologna ${ }^{b}$, Bologna, Italy

G. Abbiendi ${ }^{a}$, C. Battilana ${ }^{a, b}$, D. Bonacorsi ${ }^{a}, b$, L. Borgonovi $^{a}, b$, S. Braibant-Giacomelli ${ }^{a, b}$, L. Brigliadori ${ }^{a, b}$, R. Campanini ${ }^{a, b}$, P. Capiluppi ${ }^{a, b}$, A. Castro ${ }^{a, b}$, F.R. Cavallo $^{a}$,

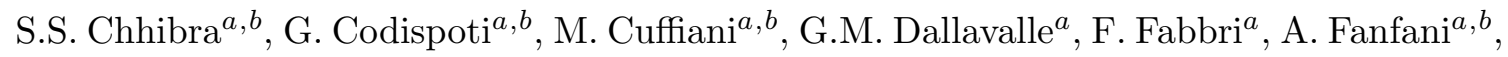
D. Fasanella ${ }^{a, b}$, P. Giacomelli ${ }^{a}$, C. Grandi ${ }^{a}$, L. Guiducci ${ }^{a}, b$, F. Iemmi, S. Marcellini ${ }^{a}$, G. Masetti ${ }^{a}$, A. Montanari ${ }^{a}$, F.L. Navarria ${ }^{a, b}$, A. Perrotta ${ }^{a}$, T. Rovelli ${ }^{a}, b$, G.P. Siroli $^{a, b}$, N. Tosi ${ }^{a}$

INFN Sezione di Catania ${ }^{a}$, Università di Catania ${ }^{b}$, Catania, Italy

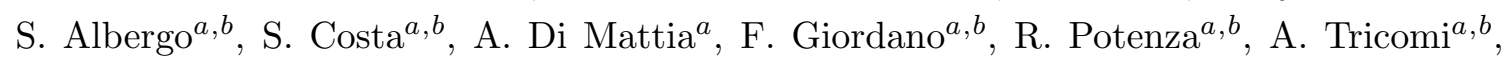
C. Tuve ${ }^{a, b}$ 
INFN Sezione di Firenze ${ }^{a}$, Università di Firenze ${ }^{b}$, Firenze, Italy

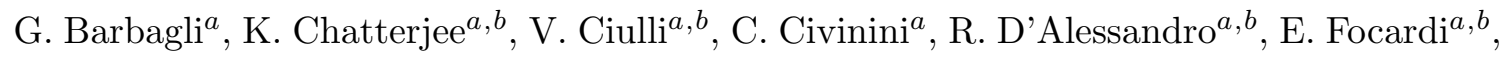
G. Latino, P. Lenzi ${ }^{a}, b$, M. Meschini ${ }^{a}$, S. Paoletti ${ }^{a}$, L. Russo $^{a}, 29$, G. Sguazzoni ${ }^{a}$, D. Strom ${ }^{a}$, L. Viliani ${ }^{a}$

INFN Laboratori Nazionali di Frascati, Frascati, Italy

L. Benussi, S. Bianco, F. Fabbri, D. Piccolo, F. Primavera ${ }^{14}$

INFN Sezione di Genova ${ }^{a}$, Università di Genova ${ }^{b}$, Genova, Italy

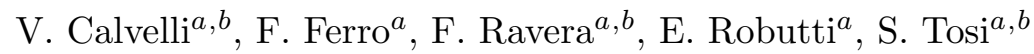

INFN Sezione di Milano-Bicocca ${ }^{a}$, Università di Milano-Bicocca ${ }^{b}$, Milano, Italy

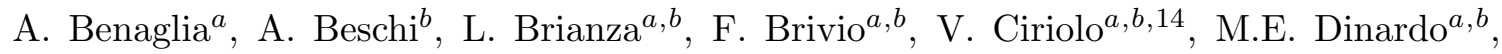
S. Fiorendi ${ }^{a, b}$, S. Gennai ${ }^{a}$, A. Ghezzi ${ }^{a, b}$, P. Govoni ${ }^{a, b}$, M. Malberti ${ }^{a, b}$, S. Malvezzi ${ }^{a}$, R.A. Manzoni ${ }^{a, b}$, D. Menasce ${ }^{a}$, L. Moroni $^{a}$, M. Paganoni $^{a}, b$, K. Pauwels $^{a}, b$, D. Pedrini $^{a}$, S. Pigazzini ${ }^{a, b, 30}$, S. Ragazzi ${ }^{a, b}$, T. Tabarelli de Fatis ${ }^{a, b}$

INFN Sezione di Napoli ${ }^{a}$, Università di Napoli 'Federico II' ${ }^{b}$, Napoli, Italy, Università della Basilicata ${ }^{c}$, Potenza, Italy, Università G. Marconi ${ }^{d}$, Roma, Italy

S. Buontempo ${ }^{a}$, N. Cavallo ${ }^{a, c}$, S. Di Guida ${ }^{a, d, 14}$, F. Fabozzi ${ }^{a, c}$, F. Fienga ${ }^{a, b}$,

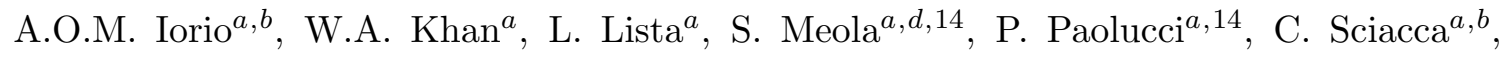
F. Thyssen ${ }^{a}$

INFN Sezione di Padova ${ }^{a}$, Università di Padova ${ }^{b}$, Padova, Italy, Università di Trento ${ }^{c}$, Trento, Italy

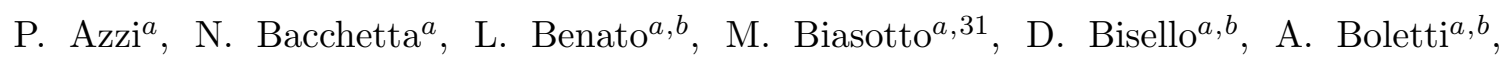
R. Carlin ${ }^{a, b}$, A. Carvalho Antunes De Oliveira ${ }^{a, b}$, P. Checchia ${ }^{a}$, M. Dall'Osso ${ }^{a, b}$, P. De Castro Manzano $^{a}$, T. Dorigo ${ }^{a}$, U. Dosselli ${ }^{a}$, F. Gasparini ${ }^{a}, b$, A. Gozzelino $^{a}$, S. Lacaprara ${ }^{a}$, P. Lujan, M. Margoni ${ }^{a, b}$, A.T. Meneguzzo ${ }^{a, b}$, N. Pozzobon ${ }^{a}, b$, P. Ronchese ${ }^{a, b},{\text { R. } \operatorname{Rossin}^{a, b}}^{,}$ F. Simonetto ${ }^{a, b}$, A. Tiko, E. Torassa ${ }^{a}$, M. Zanetti ${ }^{a, b}$, P. Zotto ${ }^{a, b}$

INFN Sezione di Pavia ${ }^{a}$, Università di Pavia ${ }^{b}$, Pavia, Italy

A. Braghieri ${ }^{a}$, A. Magnani ${ }^{a}$, P. Montagna ${ }^{a, b}$, S.P. Ratti ${ }^{a, b}, \mathrm{~V} \cdot \operatorname{Re}^{a}$, M. Ressegotti $^{a, b}$, C. Riccardi ${ }^{a}, b$, P. Salvini ${ }^{a}$, I. Vai $^{a, b}$, P. Vitulo ${ }^{a, b}$

INFN Sezione di Perugia ${ }^{a}$, Università di Perugia ${ }^{b}$, Perugia, Italy

L. Alunni Solestizi ${ }^{a, b}$, M. Biasini ${ }^{a, b}$, G.M. Bilei ${ }^{a}$, C. Cecchi $^{a, b}$, D. Ciangottini ${ }^{a, b}$, L. Fanò $^{a, b}$, P. Lariccia ${ }^{a}, b$, R. Leonardi ${ }^{a, b}$, E. Manoni $^{a}$, G. Mantovani ${ }^{a, b}$, V. Mariani $^{a}, b$, M. Menichelli $^{a}$, A. $\operatorname{Rossi}^{a, b}$, A. Santocchia ${ }^{a, b}$, D. Spiga ${ }^{a}$

INFN Sezione di Pisa ${ }^{a}$, Università di Pisa ${ }^{b}$, Scuola Normale Superiore di Pisa ${ }^{c}$, Pisa, Italy

K. Androsov ${ }^{a}$, P. Azzurri ${ }^{a}, 14$, G. Bagliesi ${ }^{a}$, L. Bianchini ${ }^{a}$, T. Boccali ${ }^{a}$, L. Borrello,

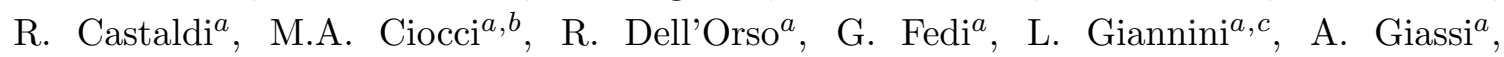
M.T. Grippo ${ }^{a, 29}$, F. Ligabue ${ }^{a, c}$, T. Lomtadze ${ }^{a}$, E. Manca ${ }^{a, c}$, G. Mandorlia ${ }^{a, c}$, 


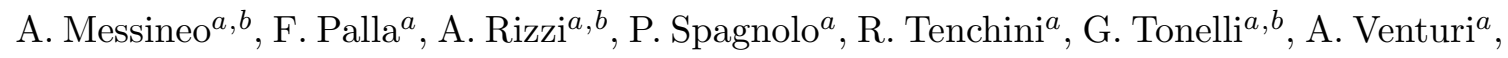
P.G. Verdini ${ }^{a}$

INFN Sezione di Roma ${ }^{a}$, Sapienza Università di Roma ${ }^{b}$, Rome, Italy

L. Barone ${ }^{a, b}$, F. Cavallari ${ }^{a}$, M. Cipriani ${ }^{a, b}$, N. Daci ${ }^{a}$, D. Del Re $e^{a, b}$ E. Di $\operatorname{Marco}^{a, b}$, M. Diemoz ${ }^{a}$, S. Gelli ${ }^{a, b}$, E. Longo ${ }^{a, b}$, F. Margaroli ${ }^{a, b}$, B. Marzocchi ${ }^{a, b}$,

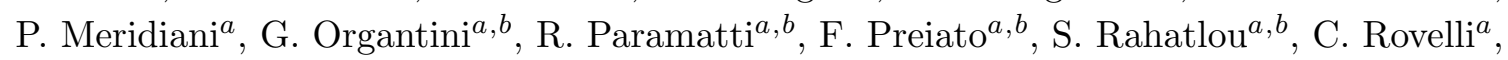
F. Santanastasio ${ }^{a, b}$

INFN Sezione di Torino ${ }^{a}$, Università di Torino ${ }^{b}$, Torino, Italy, Università del Piemonte Orientale ${ }^{c}$, Novara, Italy

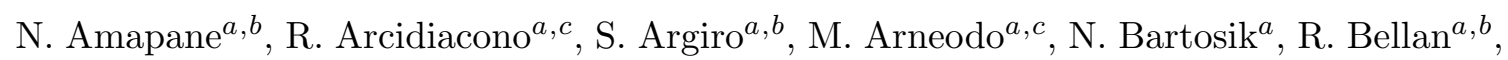
C. Biino ${ }^{a}$, N. Cartiglia ${ }^{a}$, R. Castello ${ }^{a, b}$, F. Cenna ${ }^{a, b}$, M. Costa $^{a, b}$, R. Covarelli ${ }^{a, b}$, A. Degano ${ }^{a, b}$, N. Demaria ${ }^{a}$, B. Kiani ${ }^{a, b}$, C. Mariotti ${ }^{a}$, S. Maselli ${ }^{a}$, E. Migliore ${ }^{a, b}$, V. Monaco ${ }^{a, b}$, E. Monteil ${ }^{a, b}$, M. Monteno ${ }^{a}$, M.M. Obertino ${ }^{a, b}$, L. Pacher $^{a, b}$, N. Pastrone ${ }^{a}$, M. Pelliccioni ${ }^{a}$, G.L. Pinna Angioni ${ }^{a, b}$, A. Romero ${ }^{a, b}$, M. Ruspa ${ }^{a, c}$, R. Sacchi ${ }^{a, b}$, K. Shchelina ${ }^{a, b}$, V. Sola ${ }^{a}$, A. Solano ${ }^{a, b}$, A. Staiano ${ }^{a}$, P. Traczyk ${ }^{a, b}$

INFN Sezione di Trieste ${ }^{a}$, Università di Trieste ${ }^{b}$, Trieste, Italy

S. Belforte ${ }^{a}$, M. Casarsa ${ }^{a}$, F. Cossutti ${ }^{a}$, G. Della Ricca ${ }^{a, b}$, A. Zanetti ${ }^{a}$

\section{Kyungpook National University}

D.H. Kim, G.N. Kim, M.S. Kim, J. Lee, S. Lee, S.W. Lee, C.S. Moon, Y.D. Oh, S. Sekmen, D.C. Son, Y.C. Yang

Chonnam National University, Institute for Universe and Elementary Particles, Kwangju, Korea

H. Kim, D.H. Moon, G. Oh

\section{Hanyang University, Seoul, Korea}

J.A. Brochero Cifuentes, J. Goh, T.J. Kim

Korea University, Seoul, Korea

S. Cho, S. Choi, Y. Go, D. Gyun, S. Ha, B. Hong, Y. Jo, Y. Kim, K. Lee, K.S. Lee, S. Lee, J. Lim, S.K. Park, Y. Roh

\section{Seoul National University, Seoul, Korea}

J. Almond, J. Kim, J.S. Kim, H. Lee, K. Lee, K. Nam, S.B. Oh, B.C. Radburn-Smith, S.h. Seo, U.K. Yang, H.D. Yoo, G.B. Yu

University of Seoul, Seoul, Korea

H. Kim, J.H. Kim, J.S.H. Lee, I.C. Park

\section{Sungkyunkwan University, Suwon, Korea}

Y. Choi, C. Hwang, J. Lee, I. Yu

Vilnius University, Vilnius, Lithuania

V. Dudenas, A. Juodagalvis, J. Vaitkus 
National Centre for Particle Physics, Universiti Malaya, Kuala Lumpur, Malaysia

I. Ahmed, Z.A. Ibrahim, M.A.B. Md $\mathrm{Ali}^{32}$, F. Mohamad Idris ${ }^{33}$, W.A.T. Wan Abdullah, M.N. Yusli, Z. Zolkapli

Centro de Investigacion y de Estudios Avanzados del IPN, Mexico City, Mexico Reyes-Almanza, R, Ramirez-Sanchez, G., Duran-Osuna, M. C., H. Castilla-Valdez, E. De La Cruz-Burelo, I. Heredia-De La Cruz ${ }^{34}$, Rabadan-Trejo, R. I., R. Lopez-Fernandez, J. Mejia Guisao, A. Sanchez-Hernandez

Universidad Iberoamericana, Mexico City, Mexico

S. Carrillo Moreno, C. Oropeza Barrera, F. Vazquez Valencia

Benemerita Universidad Autonoma de Puebla, Puebla, Mexico

J. Eysermans, I. Pedraza, H.A. Salazar Ibarguen, C. Uribe Estrada

Universidad Autónoma de San Luis Potosí, San Luis Potosí, Mexico

A. Morelos Pineda

University of Auckland, Auckland, New Zealand

D. Krofcheck

University of Canterbury, Christchurch, New Zealand

S. Bheesette, P.H. Butler

National Centre for Physics, Quaid-I-Azam University, Islamabad, Pakistan

A. Ahmad, M. Ahmad, Q. Hassan, H.R. Hoorani, A. Saddique, M.A. Shah, M. Shoaib, M. Waqas

National Centre for Nuclear Research, Swierk, Poland

H. Bialkowska, M. Bluj, B. Boimska, T. Frueboes, M. Górski, M. Kazana, K. Nawrocki, M. Szleper, P. Zalewski

Institute of Experimental Physics, Faculty of Physics, University of Warsaw, Warsaw, Poland

K. Bunkowski, A. Byszuk ${ }^{35}$, K. Doroba, A. Kalinowski, M. Konecki, J. Krolikowski, M. Misiura, M. Olszewski, A. Pyskir, M. Walczak

Laboratório de Instrumentação e Física Experimental de Partículas, Lisboa, Portugal

P. Bargassa, C. Beirão Da Cruz E Silva, A. Di Francesco, P. Faccioli, B. Galinhas, M. Gallinaro, J. Hollar, N. Leonardo, L. Lloret Iglesias, M.V. Nemallapudi, J. Seixas, G. Strong, O. Toldaiev, D. Vadruccio, J. Varela

Joint Institute for Nuclear Research, Dubna, Russia

S. Afanasiev, P. Bunin, M. Gavrilenko, I. Golutvin, I. Gorbunov, A. Kamenev, V. Karjavin, A. Lanev, A. Malakhov, V. Matveev ${ }^{36,37}$, P. Moisenz, V. Palichik, V. Perelygin, S. Shmatov, S. Shulha, N. Skatchkov, V. Smirnov, N. Voytishin, A. Zarubin 
Petersburg Nuclear Physics Institute, Gatchina (St. Petersburg), Russia

Y. Ivanov, V. Kim ${ }^{38}$, E. Kuznetsova ${ }^{39}$, P. Levchenko, V. Murzin, V. Oreshkin, I. Smirnov,

D. Sosnov, V. Sulimov, L. Uvarov, S. Vavilov, A. Vorobyev

Institute for Nuclear Research, Moscow, Russia

Yu. Andreev, A. Dermenev, S. Gninenko, N. Golubev, A. Karneyeu, M. Kirsanov,

N. Krasnikov, A. Pashenkov, D. Tlisov, A. Toropin

Institute for Theoretical and Experimental Physics, Moscow, Russia

V. Epshteyn, V. Gavrilov, N. Lychkovskaya, V. Popov, I. Pozdnyakov, G. Safronov, A. Spiridonov, A. Stepennov, V. Stolin, M. Toms, E. Vlasov, A. Zhokin

Moscow Institute of Physics and Technology, Moscow, Russia

T. Aushev, A. Bylinkin ${ }^{37}$

National Research Nuclear University 'Moscow Engineering Physics Institute' (MEPhI), Moscow, Russia

R. Chistov ${ }^{40}$, M. Danilov ${ }^{40}$, P. Parygin, D. Philippov, S. Polikarpov, E. Tarkovskii

P.N. Lebedev Physical Institute, Moscow, Russia

V. Andreev, M. Azarkin ${ }^{37}$, I. Dremin ${ }^{37}$, M. Kirakosyan ${ }^{37}$, S.V. Rusakov, A. Terkulov

Skobeltsyn Institute of Nuclear Physics, Lomonosov Moscow State University, Moscow, Russia

A. Baskakov, A. Belyaev, E. Boos, V. Bunichev, M. Dubinin ${ }^{41}$, L. Dudko, A. Gribushin,

V. Klyukhin, N. Korneeva, I. Lokhtin, I. Miagkov, S. Obraztsov, M. Perfilov, V. Savrin,

P. Volkov

Novosibirsk State University (NSU), Novosibirsk, Russia

V. Blinov ${ }^{42}$, D. Shtol ${ }^{42}$, Y. Skovpen ${ }^{42}$

State Research Center of Russian Federation, Institute for High Energy Physics of NRC \&quot;Kurchatov Institute\&quot;, Protvino, Russia

I. Azhgirey, I. Bayshev, S. Bitioukov, D. Elumakhov, A. Godizov, V. Kachanov, A. Kalinin,

D. Konstantinov, P. Mandrik, V. Petrov, R. Ryutin, A. Sobol, S. Troshin, N. Tyurin,

A. Uzunian, A. Volkov

National Research Tomsk Polytechnic University, Tomsk, Russia

A. Babaev

University of Belgrade, Faculty of Physics and Vinca Institute of Nuclear Sciences, Belgrade, Serbia

P. Adzic ${ }^{43}$, P. Cirkovic, D. Devetak, M. Dordevic, J. Milosevic

Centro de Investigaciones Energéticas Medioambientales y Tecnológicas (CIEMAT), Madrid, Spain

J. Alcaraz Maestre, I. Bachiller, M. Barrio Luna, M. Cerrada, N. Colino, B. De La Cruz, A. Delgado Peris, C. Fernandez Bedoya, J.P. Fernández Ramos, J. Flix, M.C. Fouz, O. Gonzalez Lopez, S. Goy Lopez, J.M. Hernandez, M.I. Josa, D. Moran, A. Pérez-Calero 
Yzquierdo, J. Puerta Pelayo, I. Redondo, L. Romero, M.S. Soares, A. Triossi, A. Álvarez Fernández

\section{Universidad Autónoma de Madrid, Madrid, Spain}

C. Albajar, J.F. de Trocóniz

\section{Universidad de Oviedo, Oviedo, Spain}

J. Cuevas, C. Erice, J. Fernandez Menendez, S. Folgueras, I. Gonzalez Caballero, J.R. González Fernández, E. Palencia Cortezon, S. Sanchez Cruz, P. Vischia, J.M. Vizan Garcia

Instituto de Física de Cantabria (IFCA), CSIC-Universidad de Cantabria, Santander, Spain

I.J. Cabrillo, A. Calderon, B. Chazin Quero, J. Duarte Campderros, M. Fernandez, P.J. Fernández Manteca, J. Garcia-Ferrero, A. García Alonso, G. Gomez, A. Lopez Virto, J. Marco, C. Martinez Rivero, P. Martinez Ruiz del Arbol, F. Matorras, J. Piedra Gomez, C. Prieels, T. Rodrigo, A. Ruiz-Jimeno, L. Scodellaro, N. Trevisani, I. Vila, R. Vilar Cortabitarte

\section{CERN, European Organization for Nuclear Research, Geneva, Switzerland}

D. Abbaneo, B. Akgun, E. Auffray, P. Baillon, A.H. Ball, D. Barney, J. Bendavid, M. Bianco, A. Bocci, C. Botta, T. Camporesi, M. Cepeda, G. Cerminara, E. Chapon, Y. Chen, D. d'Enterria, A. Dabrowski, V. Daponte, A. David, M. De Gruttola, A. De Roeck, N. Deelen, M. Dobson, T. du Pree, M. Dünser, N. Dupont, A. Elliott-Peisert, P. Everaerts, F. Fallavollita ${ }^{44}$, G. Franzoni, J. Fulcher, W. Funk, D. Gigi, A. Gilbert, K. Gill, F. Glege, D. Gulhan, J. Hegeman, V. Innocente, A. Jafari, P. Janot, O. Karacheban ${ }^{17}$, J. Kieseler, V. Knünz, A. Kornmayer, M.J. Kortelainen, M. Krammer ${ }^{1}$, C. Lange, P. Lecoq, C. Lourenço, M.T. Lucchini, L. Malgeri, M. Mannelli, A. Martelli, F. Meijers, J.A. Merlin, S. Mersi, E. Meschi, P. Milenovic ${ }^{45}$, F. Moortgat, M. Mulders, H. Neugebauer, J. Ngadiuba, S. Orfanelli, L. Orsini, F. Pantaleo ${ }^{14}$, L. Pape, E. Perez, M. Peruzzi, A. Petrilli, G. Petrucciani, A. Pfeiffer, M. Pierini, F.M. Pitters, D. Rabady, A. Racz, T. Reis, G. Rolandi ${ }^{46}$, M. Rovere, H. Sakulin, C. Schäfer, C. Schwick, M. Seidel, M. Selvaggi, A. Sharma, P. Silva, P. Sphicas ${ }^{47}$, A. Stakia, J. Steggemann, M. Stoye, M. Tosi, D. Treille, A. Tsirou, V. Veckalns ${ }^{48}$, M. Verweij, W.D. Zeuner

\section{Paul Scherrer Institut, Villigen, Switzerland}

W. Bertl ${ }^{\dagger}$, L. Caminada ${ }^{49}$, K. Deiters, W. Erdmann, R. Horisberger, Q. Ingram, H.C. Kaestli, D. Kotlinski, U. Langenegger, T. Rohe, S.A. Wiederkehr

ETH Zurich - Institute for Particle Physics and Astrophysics (IPA), Zurich, Switzerland

M. Backhaus, L. Bäni, P. Berger, B. Casal, G. Dissertori, M. Dittmar, M. Donegà, C. Dorfer, C. Grab, C. Heidegger, D. Hits, J. Hoss, T. Klijnsma, W. Lustermann, B. Mangano, M. Marionneau, M.T. Meinhard, D. Meister, F. Micheli, P. Musella, F. NessiTedaldi, F. Pandolfi, J. Pata, F. Pauss, G. Perrin, L. Perrozzi, M. Quittnat, M. Reichmann, 
D.A. Sanz Becerra, M. Schönenberger, L. Shchutska, V.R. Tavolaro, K. Theofilatos, M.L. Vesterbacka Olsson, R. Wallny, D.H. Zhu

Universität Zürich, Zurich, Switzerland

T.K. Aarrestad, C. Amsler ${ }^{50}$, D. Brzhechko, M.F. Canelli, A. De Cosa, R. Del Burgo, S. Donato, C. Galloni, T. Hreus, B. Kilminster, I. Neutelings, D. Pinna, G. Rauco, P. Robmann, D. Salerno, K. Schweiger, C. Seitz, Y. Takahashi, A. Zucchetta

National Central University, Chung-Li, Taiwan

V. Candelise, Y.H. Chang, K.y. Cheng, T.H. Doan, Sh. Jain, R. Khurana, C.M. Kuo,

W. Lin, A. Pozdnyakov, S.S. Yu

National Taiwan University (NTU), Taipei, Taiwan

Arun Kumar, P. Chang, Y. Chao, K.F. Chen, P.H. Chen, F. Fiori, W.-S. Hou, Y. Hsiung, Y.F. Liu, R.-S. Lu, E. Paganis, A. Psallidas, A. Steen, J.f. Tsai

Chulalongkorn University, Faculty of Science, Department of Physics, Bangkok, Thailand

B. Asavapibhop, K. Kovitanggoon, G. Singh, N. Srimanobhas

Çukurova University, Physics Department, Science and Art Faculty, Adana, Turkey

A. Bat, F. Boran, S. Cerci ${ }^{51}$, S. Damarseckin, Z.S. Demiroglu, C. Dozen, I. Dumanoglu,

S. Girgis, G. Gokbulut, Y. Guler, I. Hos ${ }^{52}$, E.E. Kangal ${ }^{53}$, O. Kara, A. Kayis Topaksu,

U. Kiminsu, M. Oglakci, G. Onengut, K. Ozdemir ${ }^{54}$, D. Sunar Cerci ${ }^{51}$, U.G. Tok,

H. Topakli ${ }^{55}$, S. Turkcapar, I.S. Zorbakir, C. Zorbilmez

Middle East Technical University, Physics Department, Ankara, Turkey

G. Karapinar ${ }^{56}$, K. Ocalan ${ }^{57}$, M. Yalvac, M. Zeyrek

Bogazici University, Istanbul, Turkey

E. Gülmez, M. Kaya ${ }^{58}$, O. Kaya ${ }^{59}$, S. Tekten, E.A. Yetkin ${ }^{60}$

Istanbul Technical University, Istanbul, Turkey

M.N. Agaras, S. Atay, A. Cakir, K. Cankocak, Y. Komurcu

Institute for Scintillation Materials of National Academy of Science of Ukraine, Kharkov, Ukraine

B. Grynyov

National Scientific Center, Kharkov Institute of Physics and Technology, Kharkov, Ukraine

L. Levchuk

University of Bristol, Bristol, United Kingdom

F. Ball, L. Beck, J.J. Brooke, D. Burns, E. Clement, D. Cussans, O. Davignon, H. Flacher,

J. Goldstein, G.P. Heath, H.F. Heath, L. Kreczko, D.M. Newbold ${ }^{61}$, S. Paramesvaran,

T. Sakuma, S. Seif El Nasr-storey, D. Smith, V.J. Smith 


\section{Rutherford Appleton Laboratory, Didcot, United Kingdom}

K.W. Bell, A. Belyaev ${ }^{62}$, C. Brew, R.M. Brown, L. Calligaris, D. Cieri, D.J.A. Cockerill, J.A. Coughlan, K. Harder, S. Harper, J. Linacre, E. Olaiya, D. Petyt, C.H. ShepherdThemistocleous, A. Thea, I.R. Tomalin, T. Williams, W.J. Womersley

\section{Imperial College, London, United Kingdom}

G. Auzinger, R. Bainbridge, P. Bloch, J. Borg, S. Breeze, O. Buchmuller, A. Bundock, S. Casasso, D. Colling, L. Corpe, P. Dauncey, G. Davies, M. Della Negra, R. Di Maria, Y. Haddad, G. Hall, G. Iles, T. James, M. Komm, R. Lane, C. Laner, L. Lyons, A.-M. Magnan, S. Malik, L. Mastrolorenzo, T. Matsushita, J. Nash ${ }^{63}$, A. Nikitenko ${ }^{6}$, V. Palladino, M. Pesaresi, A. Richards, A. Rose, E. Scott, C. Seez, A. Shtipliyski,

T. Strebler, S. Summers, A. Tapper, K. Uchida, M. Vazquez Acosta ${ }^{64}$, T. Virdee ${ }^{14}$, N. Wardle, D. Winterbottom, J. Wright, S.C. Zenz

Brunel University, Uxbridge, United Kingdom

J.E. Cole, P.R. Hobson, A. Khan, P. Kyberd, A. Morton, I.D. Reid, L. Teodorescu, S. Zahid Baylor University, Waco, U.S.A.

A. Borzou, K. Call, J. Dittmann, K. Hatakeyama, H. Liu, N. Pastika, C. Smith

Catholic University of America, Washington DC, U.S.A.

R. Bartek, A. Dominguez

The University of Alabama, Tuscaloosa, U.S.A.

A. Buccilli, S.I. Cooper, C. Henderson, P. Rumerio, C. West

Boston University, Boston, U.S.A.

D. Arcaro, A. Avetisyan, T. Bose, D. Gastler, D. Rankin, C. Richardson, J. Rohlf, L. Sulak, D. Zou

\section{Brown University, Providence, U.S.A.}

G. Benelli, D. Cutts, M. Hadley, J. Hakala, U. Heintz, J.M. Hogan ${ }^{65}$, K.H.M. Kwok, E. Laird, G. Landsberg, J. Lee, Z. Mao, M. Narain, J. Pazzini, S. Piperov, S. Sagir, R. Syarif, D. Yu

University of California, Davis, Davis, U.S.A.

R. Band, C. Brainerd, R. Breedon, D. Burns, M. Calderon De La Barca Sanchez, M. Chertok, J. Conway, R. Conway, P.T. Cox, R. Erbacher, C. Flores, G. Funk, W. Ko, R. Lander, C. Mclean, M. Mulhearn, D. Pellett, J. Pilot, S. Shalhout, M. Shi, J. Smith, D. Stolp, D. Taylor, K. Tos, M. Tripathi, Z. Wang, F. Zhang

\section{University of California, Los Angeles, U.S.A.}

M. Bachtis, C. Bravo, R. Cousins, A. Dasgupta, A. Florent, J. Hauser, M. Ignatenko, N. Mccoll, S. Regnard, D. Saltzberg, C. Schnaible, V. Valuev

University of California, Riverside, Riverside, U.S.A.

E. Bouvier, K. Burt, R. Clare, J. Ellison, J.W. Gary, S.M.A. Ghiasi Shirazi, G. Hanson, G. Karapostoli, E. Kennedy, F. Lacroix, O.R. Long, M. Olmedo Negrete, M.I. Paneva, W. Si, L. Wang, H. Wei, S. Wimpenny, B. R. Yates 
University of California, San Diego, La Jolla, U.S.A.

J.G. Branson, S. Cittolin, M. Derdzinski, R. Gerosa, D. Gilbert, B. Hashemi, A. Holzner, D. Klein, G. Kole, V. Krutelyov, J. Letts, M. Masciovecchio, D. Olivito, S. Padhi, M. Pieri, M. Sani, V. Sharma, S. Simon, M. Tadel, A. Vartak, S. Wasserbaech ${ }^{66}$, J. Wood, F. Würthwein, A. Yagil, G. Zevi Della Porta

University of California, Santa Barbara - Department of Physics, Santa Barbara, U.S.A.

N. Amin, R. Bhandari, J. Bradmiller-Feld, C. Campagnari, M. Citron, A. Dishaw, V. Dutta, M. Franco Sevilla, L. Gouskos, R. Heller, J. Incandela, A. Ovcharova, H. Qu, J. Richman, D. Stuart, I. Suarez, J. Yoo

California Institute of Technology, Pasadena, U.S.A.

D. Anderson, A. Bornheim, J. Bunn, J.M. Lawhorn, H.B. Newman, T. Q. Nguyen, C. Pena, M. Spiropulu, J.R. Vlimant, R. Wilkinson, S. Xie, Z. Zhang, R.Y. Zhu

Carnegie Mellon University, Pittsburgh, U.S.A.

M.B. Andrews, T. Ferguson, T. Mudholkar, M. Paulini, J. Russ, M. Sun, H. Vogel, I. Vorobiev, M. Weinberg

University of Colorado Boulder, Boulder, U.S.A.

J.P. Cumalat, W.T. Ford, F. Jensen, A. Johnson, M. Krohn, S. Leontsinis, E. Macdonald, T. Mulholland, K. Stenson, K.A. Ulmer, S.R. Wagner

Cornell University, Ithaca, U.S.A.

J. Alexander, J. Chaves, Y. Cheng, J. Chu, A. Datta, S. Dittmer, K. Mcdermott, N. Mirman, J.R. Patterson, D. Quach, A. Rinkevicius, A. Ryd, L. Skinnari, L. Soffi, S.M. Tan, Z. Tao, J. Thom, J. Tucker, P. Wittich, M. Zientek

Fermi National Accelerator Laboratory, Batavia, U.S.A.

S. Abdullin, M. Albrow, M. Alyari, G. Apollinari, A. Apresyan, A. Apyan, S. Banerjee, L.A.T. Bauerdick, A. Beretvas, J. Berryhill, P.C. Bhat, G. Bolla ${ }^{\dagger}$, K. Burkett, J.N. Butler, A. Canepa, G.B. Cerati, H.W.K. Cheung, F. Chlebana, M. Cremonesi, J. Duarte, V.D. Elvira, J. Freeman, Z. Gecse, E. Gottschalk, L. Gray, D. Green, S. Grünendahl, O. Gutsche, J. Hanlon, R.M. Harris, S. Hasegawa, J. Hirschauer, Z. Hu, B. Jayatilaka, S. Jindariani, M. Johnson, U. Joshi, B. Klima, B. Kreis, S. Lammel, D. Lincoln, R. Lipton, M. Liu, T. Liu, R. Lopes De Sá, J. Lykken, K. Maeshima, N. Magini, J.M. Marraffino, D. Mason, P. McBride, P. Merkel, S. Mrenna, S. Nahn, V. O’Dell, K. Pedro, O. Prokofyev, G. Rakness, L. Ristori, A. Savoy-Navarro ${ }^{67}$, B. Schneider, E. Sexton-Kennedy, A. Soha, W.J. Spalding, L. Spiegel, S. Stoynev, J. Strait, N. Strobbe, L. Taylor, S. Tkaczyk, N.V. Tran, L. Uplegger, E.W. Vaandering, C. Vernieri, M. Verzocchi, R. Vidal, M. Wang, H.A. Weber, A. Whitbeck, W. Wu

University of Florida, Gainesville, U.S.A.

D. Acosta, P. Avery, P. Bortignon, D. Bourilkov, A. Brinkerhoff, A. Carnes, M. Carver, D. Curry, R.D. Field, I.K. Furic, S.V. Gleyzer, B.M. Joshi, J. Konigsberg, A. Korytov, 
K. Kotov, P. Ma, K. Matchev, H. Mei, G. Mitselmakher, K. Shi, D. Sperka, N. Terentyev, L. Thomas, J. Wang, S. Wang, J. Yelton

Florida International University, Miami, U.S.A.

Y.R. Joshi, S. Linn, P. Markowitz, J.L. Rodriguez

Florida State University, Tallahassee, U.S.A.

A. Ackert, T. Adams, A. Askew, S. Hagopian, V. Hagopian, K.F. Johnson, T. Kolberg, G. Martinez, T. Perry, H. Prosper, A. Saha, A. Santra, V. Sharma, R. Yohay

Florida Institute of Technology, Melbourne, U.S.A.

M.M. Baarmand, V. Bhopatkar, S. Colafranceschi, M. Hohlmann, D. Noonan, T. Roy, F. Yumiceva

University of Illinois at Chicago (UIC), Chicago, U.S.A.

M.R. Adams, L. Apanasevich, D. Berry, R.R. Betts, R. Cavanaugh, X. Chen, O. Evdokimov, C.E. Gerber, D.A. Hangal, D.J. Hofman, K. Jung, J. Kamin, I.D. Sandoval Gonzalez, M.B. Tonjes, N. Varelas, H. Wang, Z. Wu, J. Zhang

The University of Iowa, Iowa City, U.S.A.

B. Bilki ${ }^{68}$, W. Clarida, K. Dilsiz ${ }^{69}$, S. Durgut, R.P. Gandrajula, M. Haytmyradov, V. Khristenko, J.-P. Merlo, H. Mermerkaya ${ }^{70}$, A. Mestvirishvili, A. Moeller, J. Nachtman, H. Ogul ${ }^{71}$, Y. Onel, F. Ozok ${ }^{72}$, A. Penzo, C. Snyder, E. Tiras, J. Wetzel, K. Yi

Johns Hopkins University, Baltimore, U.S.A.

B. Blumenfeld, A. Cocoros, N. Eminizer, D. Fehling, L. Feng, A.V. Gritsan, P. Maksimovic, J. Roskes, U. Sarica, M. Swartz, M. Xiao, C. You

The University of Kansas, Lawrence, U.S.A.

A. Al-bataineh, P. Baringer, A. Bean, S. Boren, J. Bowen, J. Castle, S. Khalil, A. Kropivnitskaya, D. Majumder, W. Mcbrayer, M. Murray, C. Rogan, C. Royon, S. Sanders, E. Schmitz, J.D. Tapia Takaki, Q. Wang

Kansas State University, Manhattan, U.S.A.

A. Ivanov, K. Kaadze, Y. Maravin, A. Mohammadi, L.K. Saini, N. Skhirtladze

Lawrence Livermore National Laboratory, Livermore, U.S.A.

F. Rebassoo, D. Wright

University of Maryland, College Park, U.S.A.

A. Baden, O. Baron, A. Belloni, S.C. Eno, Y. Feng, C. Ferraioli, N.J. Hadley, S. Jabeen, G.Y. Jeng, R.G. Kellogg, J. Kunkle, A.C. Mignerey, F. Ricci-Tam, Y.H. Shin, A. Skuja, S.C. Tonwar

\section{Massachusetts Institute of Technology, Cambridge, U.S.A.}

D. Abercrombie, B. Allen, V. Azzolini, R. Barbieri, A. Baty, G. Bauer, R. Bi, S. Brandt, W. Busza, I.A. Cali, M. D'Alfonso, Z. Demiragli, G. Gomez Ceballos, M. Goncharov, P. Harris, D. Hsu, M. Hu, Y. Iiyama, G.M. Innocenti, M. Klute, D. Kovalskyi, Y.-J. Lee, A. Levin, P.D. Luckey, B. Maier, A.C. Marini, C. Mcginn, C. Mironov, S. Narayanan, 
X. Niu, C. Paus, C. Roland, G. Roland, J. Salfeld-Nebgen, G.S.F. Stephans, K. Sumorok, K. Tatar, D. Velicanu, J. Wang, T.W. Wang, B. Wyslouch, S. Zhaozhong

University of Minnesota, Minneapolis, U.S.A.

A.C. Benvenuti, R.M. Chatterjee, A. Evans, P. Hansen, S. Kalafut, Y. Kubota, Z. Lesko, J. Mans, S. Nourbakhsh, N. Ruckstuhl, R. Rusack, J. Turkewitz, M.A. Wadud

University of Mississippi, Oxford, U.S.A.

J.G. Acosta, S. Oliveros

University of Nebraska-Lincoln, Lincoln, U.S.A.

E. Avdeeva, K. Bloom, D.R. Claes, C. Fangmeier, F. Golf, R. Gonzalez Suarez, R. Kamalieddin, I. Kravchenko, J. Monroy, J.E. Siado, G.R. Snow, B. Stieger

State University of New York at Buffalo, Buffalo, U.S.A.

J. Dolen, A. Godshalk, C. Harrington, I. Iashvili, D. Nguyen, A. Parker, S. Rappoccio, B. Roozbahani

Northeastern University, Boston, U.S.A.

G. Alverson, E. Barberis, C. Freer, A. Hortiangtham, A. Massironi, D.M. Morse, T. Orimoto, R. Teixeira De Lima, T. Wamorkar, B. Wang, A. Wisecarver, D. Wood

Northwestern University, Evanston, U.S.A.

S. Bhattacharya, O. Charaf, K.A. Hahn, N. Mucia, N. Odell, M.H. Schmitt, K. Sung, M. Trovato, M. Velasco

University of Notre Dame, Notre Dame, U.S.A.

R. Bucci, N. Dev, M. Hildreth, K. Hurtado Anampa, C. Jessop, D.J. Karmgard, N. Kellams, K. Lannon, W. Li, N. Loukas, N. Marinelli, F. Meng, C. Mueller, Y. Musienko ${ }^{36}$, M. Planer, A. Reinsvold, R. Ruchti, P. Siddireddy, G. Smith, S. Taroni, M. Wayne, A. Wightman, M. Wolf, A. Woodard

The Ohio State University, Columbus, U.S.A.

J. Alimena, L. Antonelli, B. Bylsma, L.S. Durkin, S. Flowers, B. Francis, A. Hart, C. Hill, W. Ji, T.Y. Ling, W. Luo, B.L. Winer, H.W. Wulsin

Princeton University, Princeton, U.S.A.

S. Cooperstein, O. Driga, P. Elmer, J. Hardenbrook, P. Hebda, S. Higginbotham, A. Kalogeropoulos, D. Lange, J. Luo, D. Marlow, K. Mei, I. Ojalvo, J. Olsen, C. Palmer, P. Piroué, D. Stickland, C. Tully

University of Puerto Rico, Mayaguez, U.S.A.

S. Malik, S. Norberg

Purdue University, West Lafayette, U.S.A.

A. Barker, V.E. Barnes, S. Das, L. Gutay, M. Jones, A.W. Jung, A. Khatiwada, D.H. Miller, N. Neumeister, C.C. Peng, H. Qiu, J.F. Schulte, J. Sun, F. Wang, R. Xiao, W. Xie

Purdue University Northwest, Hammond, U.S.A.

T. Cheng, N. Parashar 
Rice University, Houston, U.S.A.

Z. Chen, K.M. Ecklund, S. Freed, F.J.M. Geurts, M. Guilbaud, M. Kilpatrick, W. Li, B. Michlin, B.P. Padley, J. Roberts, J. Rorie, W. Shi, Z. Tu, J. Zabel, A. Zhang

University of Rochester, Rochester, U.S.A.

A. Bodek, P. de Barbaro, R. Demina, Y.t. Duh, T. Ferbel, M. Galanti, A. Garcia-Bellido, J. Han, O. Hindrichs, A. Khukhunaishvili, K.H. Lo, P. Tan, M. Verzetti

The Rockefeller University, New York, U.S.A.

R. Ciesielski, K. Goulianos, C. Mesropian

Rutgers, The State University of New Jersey, Piscataway, U.S.A.

A. Agapitos, J.P. Chou, Y. Gershtein, T.A. Gómez Espinosa, E. Halkiadakis, M. Heindl, E. Hughes, S. Kaplan, R. Kunnawalkam Elayavalli, S. Kyriacou, A. Lath, R. Montalvo, K. Nash, M. Osherson, H. Saka, S. Salur, S. Schnetzer, D. Sheffield, S. Somalwar, R. Stone, S. Thomas, P. Thomassen, M. Walker

University of Tennessee, Knoxville, U.S.A.

A.G. Delannoy, J. Heideman, G. Riley, K. Rose, S. Spanier, K. Thapa

Texas A\&M University, College Station, U.S.A.

O. Bouhali ${ }^{73}$, A. Castaneda Hernandez ${ }^{73}$, A. Celik, M. Dalchenko, M. De Mattia, A. Delgado, S. Dildick, R. Eusebi, J. Gilmore, T. Huang, T. Kamon ${ }^{74}$, R. Mueller, Y. Pakhotin, R. Patel, A. Perloff, L. Perniè, D. Rathjens, A. Safonov, A. Tatarinov

Texas Tech University, Lubbock, U.S.A.

N. Akchurin, J. Damgov, F. De Guio, P.R. Dudero, J. Faulkner, E. Gurpinar, S. Kunori, K. Lamichhane, S.W. Lee, T. Mengke, S. Muthumuni, T. Peltola, S. Undleeb, I. Volobouev, Z. Wang

Vanderbilt University, Nashville, U.S.A.

S. Greene, A. Gurrola, R. Janjam, W. Johns, C. Maguire, A. Melo, H. Ni, K. Padeken, P. Sheldon, S. Tuo, J. Velkovska, Q. Xu

University of Virginia, Charlottesville, U.S.A.

M.W. Arenton, P. Barria, B. Cox, R. Hirosky, M. Joyce, A. Ledovskoy, H. Li, C. Neu, T. Sinthuprasith, Y. Wang, E. Wolfe, F. Xia

Wayne State University, Detroit, U.S.A.

R. Harr, P.E. Karchin, N. Poudyal, J. Sturdy, P. Thapa, S. Zaleski

University of Wisconsin - Madison, Madison, WI, U.S.A.

M. Brodski, J. Buchanan, C. Caillol, D. Carlsmith, S. Dasu, L. Dodd, S. Duric, B. Gomber, M. Grothe, M. Herndon, A. Hervé, U. Hussain, P. Klabbers, A. Lanaro, A. Levine, K. Long, R. Loveless, V. Rekovic, T. Ruggles, A. Savin, N. Smith, W.H. Smith, N. Woods

$\dagger$ : Deceased

1: Also at Vienna University of Technology, Vienna, Austria 
2: Also at IRFU, CEA, Université Paris-Saclay, Gif-sur-Yvette, France

3: Also at Universidade Estadual de Campinas, Campinas, Brazil

4: Also at Federal University of Rio Grande do Sul, Porto Alegre, Brazil

5: Also at Université Libre de Bruxelles, Bruxelles, Belgium

6: Also at Institute for Theoretical and Experimental Physics, Moscow, Russia

7: Also at Joint Institute for Nuclear Research, Dubna, Russia

8: Also at Cairo University, Cairo, Egypt

9: Also at Helwan University, Cairo, Egypt

10: Now at Zewail City of Science and Technology, Zewail, Egypt

11: Also at Department of Physics, King Abdulaziz University, Jeddah, Saudi Arabia

12: Also at Université de Haute Alsace, Mulhouse, France

13: Also at Skobeltsyn Institute of Nuclear Physics, Lomonosov Moscow State University, Moscow, Russia

14: Also at CERN, European Organization for Nuclear Research, Geneva, Switzerland

15: Also at RWTH Aachen University, III. Physikalisches Institut A, Aachen, Germany

16: Also at University of Hamburg, Hamburg, Germany

17: Also at Brandenburg University of Technology, Cottbus, Germany

18: Also at MTA-ELTE Lendület CMS Particle and Nuclear Physics Group, Eötvös Loránd University, Budapest, Hungary

19: Also at Institute of Nuclear Research ATOMKI, Debrecen, Hungary

20: Also at Institute of Physics, University of Debrecen, Debrecen, Hungary

21: Also at Indian Institute of Technology Bhubaneswar, Bhubaneswar, India

22: Also at Institute of Physics, Bhubaneswar, India

23: Also at Shoolini University, Solan, India

24: Also at University of Visva-Bharati, Santiniketan, India

25: Also at University of Ruhuna, Matara, Sri Lanka

26: Also at Isfahan University of Technology, Isfahan, Iran

27: Also at Yazd University, Yazd, Iran

28: Also at Plasma Physics Research Center, Science and Research Branch, Islamic Azad University, Tehran, Iran

29: Also at Università degli Studi di Siena, Siena, Italy

30: Also at INFN Sezione di Milano-Bicocca; Università di Milano-Bicocca, Milano, Italy

31: Also at Laboratori Nazionali di Legnaro dell'INFN, Legnaro, Italy

32: Also at International Islamic University of Malaysia, Kuala Lumpur, Malaysia

33: Also at Malaysian Nuclear Agency, MOSTI, Kajang, Malaysia

34: Also at Consejo Nacional de Ciencia y Tecnología, Mexico city, Mexico

35: Also at Warsaw University of Technology, Institute of Electronic Systems, Warsaw, Poland

36: Also at Institute for Nuclear Research, Moscow, Russia

37: Now at National Research Nuclear University 'Moscow Engineering Physics Institute' (MEPhI), Moscow, Russia

38: Also at St. Petersburg State Polytechnical University, St. Petersburg, Russia

39: Also at University of Florida, Gainesville, U.S.A.

40: Also at P.N. Lebedev Physical Institute, Moscow, Russia

41: Also at California Institute of Technology, Pasadena, U.S.A.

42: Also at Budker Institute of Nuclear Physics, Novosibirsk, Russia

43: Also at Faculty of Physics, University of Belgrade, Belgrade, Serbia

44: Also at INFN Sezione di Pavia; Università di Pavia, Pavia, Italy

45: Also at University of Belgrade, Faculty of Physics and Vinca Institute of Nuclear Sciences, 
Belgrade, Serbia

46: Also at Scuola Normale e Sezione dell'INFN, Pisa, Italy

47: Also at National and Kapodistrian University of Athens, Athens, Greece

48: Also at Riga Technical University, Riga, Latvia

49: Also at Universität Zürich, Zurich, Switzerland

50: Also at Stefan Meyer Institute for Subatomic Physics (SMI), Vienna, Austria

51: Also at Adiyaman University, Adiyaman, Turkey

52: Also at Istanbul Aydin University, Istanbul, Turkey

53: Also at Mersin University, Mersin, Turkey

54: Also at Piri Reis University, Istanbul, Turkey

55: Also at Gaziosmanpasa University, Tokat, Turkey

56: Also at Izmir Institute of Technology, Izmir, Turkey

57: Also at Necmettin Erbakan University, Konya, Turkey

58: Also at Marmara University, Istanbul, Turkey

59: Also at Kafkas University, Kars, Turkey

60: Also at Istanbul Bilgi University, Istanbul, Turkey

61: Also at Rutherford Appleton Laboratory, Didcot, United Kingdom

62: Also at School of Physics and Astronomy, University of Southampton, Southampton, United Kingdom

63: Also at Monash University, Faculty of Science, Clayton, Australia

64: Also at Instituto de Astrofísica de Canarias, La Laguna, Spain

65: Also at Bethel University, ST. PAUL, U.S.A.

66: Also at Utah Valley University, Orem, U.S.A.

67: Also at Purdue University, West Lafayette, U.S.A.

68: Also at Beykent University, Istanbul, Turkey

69: Also at Bingol University, Bingol, Turkey

70: Also at Erzincan University, Erzincan, Turkey

71: Also at Sinop University, Sinop, Turkey

72: Also at Mimar Sinan University, Istanbul, Istanbul, Turkey

73: Also at Texas A\&M University at Qatar, Doha, Qatar

74: Also at Kyungpook National University, Daegu, Korea 